\title{
Backstepping control with fixed-time prescribed performance for fixed wing UAV under model uncertainties and external disturbances
}

\author{
Jian Tan ${ }^{\mathrm{a}}$, Shijun Guo ${ }^{\mathrm{b}}$ \\ ${ }^{a}$ Guangdong Institute of Aeronautics and Astronautics Equipment \& Technology, Zhu Hai, \\ The People's Republic of China; ${ }^{\mathrm{b}}$ Centre for Aeronautics, Aerospace, Cranfield University, \\ Cranfield, England, UK \\ Received 05 Feb 2020, Revised 01 Aug 2020, Accepted 28 Sep 2020

\section{ARTICLE HISTORY} \\ Compiled October 1, 2020
}

\begin{abstract}
In this paper, a novel backstepping control scheme with fixed-time prescribed performance is proposed for the longitudinal model of fixed wing UAV subject to model uncertainties and external disturbances. The novel performance function with arbitrarily preassigned fixed-time convergence property is developed, which imposes priori performance envelops on both altitude and airspeed tracking errors. By using error transformed technology, the constrained fixed-time performance envelops are changed into unconstrained equivalent errors. Based on modified error compensation mechanism, a novel backstepping approach is proposed to guarantee altitude tracking equivalent error converges to the specified small neighborhood and presents excellent robustness against model uncertainties and external disturbances, and airspeed controller with fixed-time prescribed performance is designed. The proposed methodology guarantees the transient and steady-state performance of altitude and airspeed tracking errors within constrained fixed-time performance envelops in spite of lumped disturbances. Finally, numerical simulations are used to verify the effectiveness of the proposed control scheme.
\end{abstract}

\section{KEYWORDS}

UAV; prescribed performance, fixed-time control; backstepping control

\section{Introduction}

Lately, unmanned aerial vehicles (UAVs) are popular autonomous platforms for various flying missions ranging from exploration, surveillance, fire-fighting, traffic monitoring, weather research, rescue of people, power lines and oil pipelines inspection (Castañeda, Salas-Peña, \& de León-Morales, 2017). The remarkable military and commercial values of UAV render them a research hotspot (Al Younes, Drak, Noura, Rabhi, \& El Hajjaji, 2016; Mofid \& Mobayen, 2018; Poultney, Gong, \& Ashrafiuon, 2019). Therefore, it is important to design robust and high-performance control strategy with fast convergence characteristic for improving the reliability and safety of the UAV control system. During the past decades, various types of finite-time (Mobayen, 2015; Pukdeboon \& Siricharuanun, 
2014; Sun, Yun, \& Li, 2017; Xi, Mobayen, Ren, \& Jafari, 2018) and fixedtime (Espitia, Polyakov, Efimov, \& Perruquetti, 2019; Gao \& Guo, 2020; Polyakov, Efimov, \& Brogliato, 2019) control methods have been developed for various nonlinear systems. In Haghighi and Mobayen (2018), a novel adaptive terminal sliding mode control scheme is proposed to realize the finite-time convergence for both subsystems of the fourth-order system. In the pioneering work of fixed-time control (Polyakov, 2012), two fixed-time control algorithms developed by Polyakov provide fixed-time stability of the closed-loop systems independent of initial conditions. In Gao and Guo (2019), a novel fixed-time command filtered backstepping control scheme is proposed for surface vehicle, which ensures the trajectory tracking control can be achieved in fixed-time. Comprehensive analysis of the above control methods, although all have the characteristics of rapid convergence, but for certain flight phases of fixed wing UAVs, such as formation flight and landing phase, the flight control system needs to achieve high-performance precise tracking control of altitude and airspeed command with satisfactory transient and steady-state performance in specified fixed-time. The longitudinal dynamical model of the fixed wing UAV is featured with strong couplings, serious nonlinearities and high uncertainties. Actually, it is a challenging task to ensure that the altitude and airspeed tracking of the fixed wing UAV meet the prescribed transient and steady-state performance in fixed-time.

Due to the flexibility, systematic design process and good extensibility (Nikdel, Badamchizadeh, Azimirad, \& Nazari, 2016), the backstepping control is a commonly used method in the field on contro of aircraft and especially UAV (Chen, Jiang, Zhang, Jiang, \& Tao, 2016). Based on the Lyapunov stability theory, the conventional backstepping control provides a gradually recursive method to design virtual control signal from the top to the bottom, and the actual control law is designed at the last step to make the whole closed-loop system stable. In this process, the virtual control signals need to be repeatedly differentiated. As the system order and model complexity increase, the control law becomes more and more complicated, which leads to the phenomenon of "explosion of complexity" (Swaroop, Hedrick, Yip, \& Gerdes, 2000; J. Yu, Shi, \& Zhao, 2018). To address the above issue, the dynamic surface control (DSC) approach has been proposed by introducing a first-order filter in each step of the design process (Qiao, Meng, Wang, Ke, \& Sun, 2019; Qiu, Liang, Dai, Cao, \& Chen, 2015; Tong, Sui, \& Li, 2014; J. Yu, Shi, Dong, \& Yu, 2015). Command filtered backstepping control is another improved backstepping control method, which obtains the derivative of virtual control signal through command filter, and introduces error compensation mechanism to reduce the influence of the errors caused by the command filters (Farrell, Polycarpou, Sharma, \& Dong, 2009). However, most of the backstepping control methods and error compensation mechanisms are all asymptotically stable, which means that infinite time is required to achieve closed-loop system stability (J. Yu, Zhao, Yu, Lin, \& Dong, 2017). As an effective strategy to solve the tracking control problems of high-order nonlinear systems, how to ensure the finite-time convergence of the command filtered backstepping control is an important and challenging task.

It should be pointed out that the backstepping control methods are not direct and fast enough to handle the effect of the disturbances. In order to enhance the robustness of the control system, the neuro/fuzzy technique is combined with command filtered backstepping control to eliminate the influence of uncertain nonlinearities by employing neuro/fuzzy approximating structures (Li \& Tong, 2016; Peydayesh, Arefi, \& Modares, 2018). However, as extra adaptive parameters have to 
be updated, the introduction of neuro/fuzzy approximating structures increases the complexity and extra calculations of the backstepping control schemes, thus making their implementation difficult in engineering practice (Bechlioulis \& Rovithakis, 2016). On disturbance rejection tracking control problem, active anti-disturbance control based on disturbance observer has an inherent superiority in handling uncertainties (Wang, Yu, Li, \& Liu, 2019). That is, according to known model and dynamic information of system, the disturbance observer is constructed to estimate and compensate the uncertain disturbances. Due to its powerful disturbance rejection capability, active anti-disturbance control methods based on disturbance observer have been utilized for many nonlinear systems with disturbances (Back \& Kim, 2015; Besnard, Shtessel, \& Landrum, 2012; Gao \& Guo, 2020; Lu, Liu, Guo, \& Chen, 2015).

Although the above control strategies provide excellent results in terms of reference trajectory tracking performance, it is worth noting that all of them can only guarantee the stability of the system. Unfortunately, the prescribed transient performance (overshoot and convergence rate of the tracking errors) and steady tracking accuracy are difficult to guarantee due to the lack of suitable tools. As the pioneering work to handle this problem, prescribed performance control (PPC) proposed by Bechlioulis and Rovithakis is an effective way (Bechlioulis, Doulgeri, \& Rovithakis, 2012; Bechlioulis \& Rovithakis, 2014). By designing decaying performance function, PPC framework can quantitatively characterize both transient and steadystate performance of the controlled systems. Due to the prominent advantages, PPC methods attract extensive attention in application of various nonlinear systems (Bu, 2018; Tooranjipour, Vatankhah, \& Arefi, 2019), and a few PPC methods are presented to solve the flight control problem. In $\mathrm{Bu}, \mathrm{Wu}, \mathrm{Huang}$, and Wei (2016), a novel estimation-free prescribed performance backstepping controller is constructed directly using a non-affine model of air-breathing hypersonic vehicle. In Hua, Chen, and Guan (2018), for the quadrotor UAVs with time-varying payloads and wind gust disturbances, a robust adaptive prescribed performance controller is proposed. In Y. Zhang, Wang, Chang, and Wu (2019), for the carrier-based UAV subject to uncertainties, input constraints and unknown disturbances, adaptive constrained backstepping controller with prescribed performance methodology is proposed.

Although the transient performance of the convergence process can be specified in advance, however, the conventional PPC methods usually use exponentially decaying performance functions (Hua et al., 2018; Kostarigka, Doulgeri, \& Rovithakis, 2013), which can only guarantee the system states converge at the exponential rate, in other words, the system states converge to its prescribed arbitrarily small residual set only when time approaches positive infinity. For the flight control system of UAV, it is usually expected that the system states reach target commands in fixed-time. Therefore, novel fixed-time PPC framework needs to be explored to overcome the shortcomings of conventional PPC methods.

Motivated by the above discussions, in this paper, we aim at achieving arbitrarily fixed-time prescribed performance control for the longitudinal model of fixed wing UAV subject to model uncertainties and external disturbances. Compared to the existing research results, the main contributions of this paper are summarized as follows:

(1) The proposed fixed-time performance function converges to any expected tracking accuracy at arbitrarily preassigned fixed-time, with which the preassigned fixed-time performance envelops are strictly constructed for altitude and airspeed tracking errors. 
(2) In order to quickly approach the final value, the conventional PPC usually chooses large exponential convergence rate, which cause the magnitude of control input to be large or even saturation at the initial time. Compared with $\mathrm{PPC}$, the magnitude of control input under fixed-time prescribed performance control (FTPPC) at the initial time is small, which can effectively avoid control input saturation at the initial time.

(3) A novel fixed-time control scheme is proposed based on the fixed-time prescribed performance methodology and backstepping control. By using error transformed technology, the equivalent error system is stabilized based on backstepping approach, which ensures the transient and steady-state performance of tracking error within constrained fixed-time performance envelops. The novel fixed-time control scheme of this paper not only guarantee the stability of the system in fixed-time, but also meet the prescribed transient and steady-state performance.

The structure of the paper is organized as follows. Section 2 gives the longitudinal model of fixed wing UAV and reviews necessary preliminary knowledges throughout the paper. In Section 3, the novel performance function with arbitrarily preassigned fixed-time convergence property is developed, and the equivalent error transformation technology for fixed-time prescribed performance control is introduced. In Section 4 , a novel backstepping altitude controller with fixed-time prescribed performance is proposed, and airspeed controller with fixed-time prescribed performance is designed in Section 5. Section 6 uses two simulation examples to verify the effectiveness of the proposed control scheme. Section 7 gives some conclusions.

\section{Problem formulation and necessary preliminaries}

\subsection{Model description}

The longitudinal dynamical model of fixed wing UAV can be formulated as follows:

$$
\begin{aligned}
\hat{\gamma} & =V \frac{T \cos \alpha-D}{m}-g \sin \gamma \\
\dot{\gamma} & =\frac{L+T \sin \alpha}{m V}-\frac{g}{V} \cos \gamma \\
\dot{\alpha} & =q-\dot{\gamma} \\
\dot{q} & =\frac{M}{I_{y y}}
\end{aligned}
$$

The above longitudinal model of UAV is composed of five states (i.e., airspeed $V$, altitude $h$, flight-path angle $\gamma$, angle of attack $\alpha$, and pitch rate $q$ ), which are available for measurement and control law design. $m$ represents the mass of the UAV; $I_{y y}$ represents the moment of inertia; $g$ denotes the gravity acceleration. The engine thrust $T=T_{\max } \delta_{T}$ acting on the UAV is along the body axis; $T_{\max }$ and $\delta_{T}$ denote maximum of the engine thrust force and instantaneous thrust throttle setting, respectively. The aerodynamic force/torque acting on UAV are synthesized as drag component $D$, lift component $L$ and pitch moment component $M$. The aerodynamics force and torque of the UAV can be calculated from the dimensionless aerodynamic coefficients and 
have the following expressions

$$
\begin{aligned}
& L=0.5 \rho V^{2} S C_{L} \\
& D=0.5 \rho V^{2} S C_{D} \\
& M=0.5 \rho V^{2} S c C_{m}
\end{aligned}
$$

where $C_{L}, C_{D}, C_{m}$ denote lift coefficient, drag coefficient and pitching moment coefficient, respectively. $\rho$ is the air density. The reference area $S$ and the mean aerodynamic chord of the wing $c$ are considered as constant parameters. The dimensionless aerodynamic coefficients in the force/torque expressions can be decomposed in the following equations

$$
\begin{aligned}
& C_{L}=C_{L \alpha} \alpha+C_{L 0}+C_{L \delta_{e}} \delta_{e} \\
& C_{D}=C_{D \alpha^{2}} \alpha^{2}+C_{D \alpha} \alpha+C_{D 0} \\
& C_{m}=C_{m \alpha} \alpha+C_{m 0}+\frac{c q}{2 V} C_{m q}+C_{m \delta_{e}} \delta_{e}
\end{aligned}
$$

where $\delta_{e}$ denotes the elevator deflection. Other related variables and vehicle parameters can be found in $\mathrm{Z}$. Yu, Qu, and Zhang (2018). Therefore, the longitudinal model has two control inputs, throttle setting $\delta_{T}$ and elevator deflection $\delta_{e}$, and the outputs to be controlled are selected as $y=[V, h]^{T}$. The control objective of this study is to design a control scheme to guarantee altitude and airspeed tracking errors within constrained fixed-time performance envelops in spite of lumped disturbances.

Remark 1: From equations (1)-(5), it can be seen that the airspeed of the UAV is controlled by the thrust throttle setting and the altitude is controlled by the elevator. Therefore, equation (1) can be defined as the airspeed subsystem, and equations (2)(5) are defined as the altitude subsystem.

\subsection{Necessary preliminaries}

Consider the robust/exact first-order differentiator (Angulo, Moreno, \& Fridman, 2013) as follows

$$
\begin{aligned}
& \dot{\psi}_{1}=-k_{1} \vartheta \operatorname{sig}\left(\psi_{1}-x_{d}\right)^{1 / 2}-k_{3}(1-\vartheta) \operatorname{sig}\left(\psi_{1}-x_{d}\right)^{(2+\tau) / 2}+\psi_{2} \\
& \dot{\psi}_{2}=-k_{2} \vartheta \operatorname{sign}\left(\psi_{1}-x_{d}\right)-k_{4}(1-\vartheta) \operatorname{sig}\left(\psi_{1}-x_{d}\right)^{1+\tau}
\end{aligned}
$$

where sig(.) $)^{\tau}=\left.\lambda \cdot\right|^{\tau} \operatorname{sign}(\cdot), \operatorname{sign}(\cdot)$ is the standard sign function. $x_{d}$ is the input signal. $k_{1}, k_{2}$ are selected based on the original first-order sliding mode differentiator (Levant, 2003) $k_{3}, k_{4}$ are selected as $k_{3}>0, k_{4}>0$ and $\tau$ is chosen small enough, the function $\vartheta:[0, \infty) \rightarrow\{0,1\}$ is selected as

$$
\vartheta(t)= \begin{cases}0, & T \leq T_{\vartheta} \\ 1, & T>T_{\vartheta}\end{cases}
$$

with some arbitrarily chosen $T_{\vartheta}>0$. Then the following lemmas hold:

Lemma 1 (Angulo et al., 2013): The parameter $\tau>0$ is sufficiently small and $k_{1}, k_{2}, k_{3}, k_{4}$ being properly chosen, the following equalities will be established in the 
absence of input noises after finite-time transient process.

$$
\psi_{1}=x_{d}, \psi_{2}=\dot{x}_{d}
$$

Note that Lemma 1 is given provided that the input of differentiator is not affected by noise, if the input is affected by noise, then we have the following lemma.

Lemma 2 (Angulo et al., 2013): When the input signal $x_{d}$ is measured with additive noise uniformly bounded satisfying $\left|x_{d}-x_{d 0}\right|<\iota$, then the following accuracy is obtained after finite-time.

$$
\left|\psi_{1}-x_{d}\right|=0\left(L^{1 / 3} \iota^{2 / 3}\right)=\nu
$$

Lemma 3 (Zuo, 2015): For any nonnegative real numbers $\xi_{i} \geq 0, i=1, \ldots, N$ and $0<p<1$. Then

$$
\sum_{i=1}^{N} \xi_{i}^{p} \geq\left(\sum_{i=1}^{N} \xi_{i}\right)^{p}
$$

Lemma 4 (Mobayen \& Ma, 2018): Consider the continuously differentiable bounded positive definite function $V(t)$, the following inequality exists

$$
\dot{V}(t) \leq-\lambda_{1} V-\lambda_{2} V^{\gamma}
$$

where $\lambda_{1}>0, \lambda_{2}>0,0<\gamma<1$, then, the equilibrium point of system is globally finite-time stable with the settling time upper bounded by

$$
T_{r} \leq \frac{1}{\lambda_{1}(1-\gamma)} \ln \frac{\lambda_{1} V(0)^{1-\gamma}+\lambda_{2}}{\lambda_{2}}
$$

\section{Fixed-time prescribed performance control}

\subsection{Novel fixed-time performance function design}

In the conventional PPC, the exponentially decaying performance function is usually used to construet the performance envelopes. Therefore, the tracking error converges to the steady-state error bounds only when time approaches positive infinity. In practical engineering, the system states are expected to reach the control command in fixedtime in order to meet specific control requirements. In this section, a novel fixed-time performance function is presented. Inspired by the definition of exponentially decaying performance function in Bechlioulis and Rovithakis (2008), the definition of the fixed-time performance function is given as follows.

Definition 1: For a fixed-time performance function $\sigma(t)$, the following generalized properties hold.

(1) $\sigma(t)$ is $C^{n}(n \geq 1)$ continuous function on $[0,+\infty)$.

(2) $\sigma(t)$ is positive and non-increasing function from initial value $\sigma(0)=\sigma_{0}>0$ to terminal value $\sigma(T)=\sigma_{\infty}>0$, where $\sigma_{0}>\sigma_{\infty}$ are positive constants, $0<T<+\infty$ is arbitrarily preassigned fixed-time.

(3) $\dot{\sigma}(T)=0$.

(4) When $t>T, \sigma(t)=\sigma_{\infty}$. 
To better illustrate the above properties, a detailed example of fixed-time performance function is presented, i.e.

$$
\sigma(t)= \begin{cases}\left(\sigma_{0}-\sigma_{\infty}\right)\left(\frac{\sin (2 \pi t / T)}{2 \pi}-\frac{t}{T}\right)+\sigma_{0}, & 0 \leq t \leq T \\ \sigma_{\infty}, & t>T\end{cases}
$$

where $\sigma_{0}>\sigma_{\infty}>0$ are the initial value and terminal value of $\sigma(t)$, respectively. The fixed-time reachability and its $C^{2}$ continuity are given by the following property:

Property 1: The fixed-time performance function (19) is positive, non-increasing and $C^{2}$ continuous function for any given fixed-time $T$ and satisfies

$$
\sigma(t)=\sigma_{\infty}, t \geq T
$$

Proof: Take first-order and second-order time derivative of $\sigma(t)$ yield that

$$
\begin{aligned}
& \dot{\sigma}(t)= \begin{cases}\frac{\sigma_{0}-\sigma_{\infty}}{T}(\cos (2 \pi t / T)-1), & 0 \leq t \leq T \\
0, & t>T\end{cases} \\
& \ddot{\sigma}(t)=\left\{\begin{array}{ll}
-\frac{2 \pi\left(\sigma_{0}-\sigma_{\infty}\right)}{T^{2}} \sin (2 \pi t / T), & 0 \leq t \leq T \\
0, & t>T
\end{array}\right)
\end{aligned}
$$

Obviously, $\dot{\sigma}(t) \leq 0$, for $t \geq 0$, and $\sigma(t)=\sigma_{\infty}$, for $t \geq T$, so the fixed-time performance function (19) is positive and non-increasing. Since $\dot{\sigma}(T)=\ddot{\sigma}(T)=0$, the fixed-time performance function (19) is $C^{2}$ continuous function for $t \in[0,+\infty)$.

\subsection{Equivalent error transformation}

By fixed-time prescribed performance, it is meant that the tracking error $e(t)$ evolves strictly within predefined decaying envelops which converge to any expected tracking accuracy at arbitrarily preassigned fixed-time. For $\forall t \geq 0$, the mathematical expression of fixed-time prescribed performance can be formulated by the following inequality

$$
-\delta_{\min } \sigma(t) \leq e(t) \leq \delta_{\max } \sigma(t)
$$

where $\delta_{\min }>0, \delta_{\max }>0$ are positive design parameters, and satisfy $-\delta_{\min } \sigma(0) \leq$ $e(0) \leq \delta_{\text {max }} \sigma(0)$, the fixed-time performance function $\sigma(t)$ is defined in (19).

It is difficult to devise the controller directly based on prescribed performance inequality (23), the inequality constraint needs to be converted into an equality constraint. Thus, we introduce an equivalent error transformation for the tracking error $e(t)$ and performance function $\sigma(t)$ as follows

$$
e(t)=\sigma(t) s(\xi(t))
$$

where $\xi(t)$ is the transformed error, and $s(\xi):(-\infty,+\infty) \rightarrow\left(-\delta_{\min }, \delta_{\max }\right)$ is the strictly increasing smooth invertible function as follows

$$
s(\xi)=\frac{\delta_{\max } e^{\xi}-\delta_{\min } e^{-\xi}}{e^{\xi}+e^{-\xi}}
$$


From the definition of $s(\xi(t))$ and (24), it can be known that

$$
\xi(t)=s^{-1}\left(\frac{e(t)}{\sigma(t)}\right)=\frac{1}{2} \ln \frac{\delta_{\min } \sigma(t)+e(t)}{\delta_{\max } \sigma(t)-e(t)}
$$

From (26), the desired reference signal of transformed error $\xi(t)$ is

$$
\xi_{c}=\frac{1}{2} \ln \frac{\delta_{\min }}{\delta_{\max }}
$$

Take the derivative of (26), yields

$$
\begin{aligned}
& \dot{\xi}(t)=\frac{1}{2}\left(\frac{1}{e(t)+\delta_{\min } \sigma(t)}-\frac{1}{e(t)-\delta_{\max } \sigma(t)}\right)\left(\dot{e}(t)-\frac{\dot{\sigma}(t) e(t)}{\sigma(t)}\right) \\
& =\mu\left(\dot{e}(t)-\frac{\dot{\sigma}(t) e(t)}{\sigma(t)}\right)
\end{aligned}
$$

where

$$
\left.\mu=\frac{1}{2}\left(\frac{1}{e(t)+\delta_{\min } \sigma(t)}-\frac{1}{e(t)-\delta_{\max } \sigma(t)}\right)\right)
$$

Remark 2: By using error transformed technology, the constrained fixed-time performance envelops are transformed into unconstrained equivalent error. Therefore, the controller can be designed using transformed error $\xi(t)$ instead of tracking error $e(t)$.

\subsection{Comparison with conventional exponentially decaying PPC}

Following the idea of Benchlioulis and Rovithakis (Bechlioulis et al., 2012; Bechlioulis \& Rovithakis, 2014), for $\forall t \geq 0$, the conventional exponentially decaying performance function $(\mathrm{PPF}) \sigma(t)$ is chosen as

$$
\vec{\sigma}(t)=\left(\bar{\sigma}_{0}-\bar{\sigma}_{\infty}\right) e^{-l t}+\bar{\sigma}_{\infty}
$$

where $\bar{\sigma}_{0}, \bar{\sigma}_{\infty}, l$ are positive design parameters, $\bar{\sigma}_{0}=\bar{\sigma}(0)$ is selected such that $\bar{\sigma}_{0}>$ $\bar{\sigma}_{\infty}>0$.

The difference between PPF and final value has the following expression

$$
\Delta \bar{\sigma}(t)=\bar{\sigma}(t)-\bar{\sigma}_{\infty}=\left(\bar{\sigma}_{0}-\bar{\sigma}_{\infty}\right) e^{-l t}
$$

From (31), when $t=T$, we have

$$
\Delta \bar{\sigma}(T)=\left(\bar{\sigma}_{0}-\bar{\sigma}_{\infty}\right) e^{-l T}
$$

where $0<T<+\infty$ is arbitrarily preassigned fixed-time defined in (19).

The simulation comparison results of conventional exponentially decaying PPF (30) and FTPPF (19) proposed in this paper are shown in Fig. 1 with $\sigma_{0}=\bar{\sigma}_{0}=1, \sigma_{\infty}=$ $\bar{\sigma}_{\infty}=0.1, T=3 s$, and $l=0.4$ for PPF $1, l=0.7$ for PPF $2, l=1$ for PPF 3 , respectively. That is, the initial value and final value of the two performance functions are the same. 


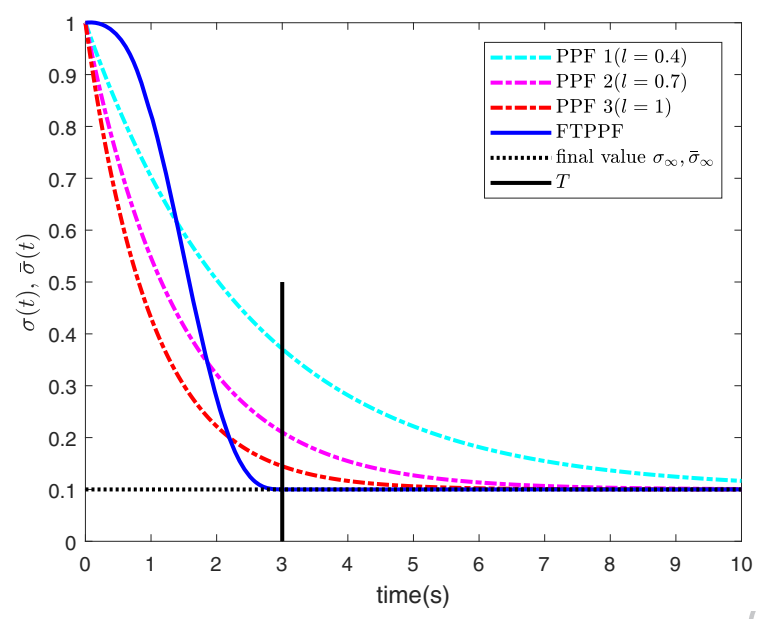

Figure 1. The comparison of conventional exponentially decaying PPF and FTPPF.

It can be seen from Fig. 1 that FTPPF converges to the final yalue in fixed-time T. From (32) and Fig. 1, as exponent $l$ increases, the differences between PPF 1-3 and final value become smaller, and only when exponent $l$ approaches positive infinity, the difference $\Delta \bar{\sigma}(T)$ can approach zero. It is worth noting that if the conventional PPC chooses large exponent $l$ in order to quickly approach the final value, the absolute value of $\lim _{t \rightarrow 0^{+}} \dot{\bar{\sigma}}(t)=-l\left(\bar{\sigma}_{0}-\bar{\sigma}_{\infty}\right)$ is large. From (28), when conventional exponentially decaying PPF $\bar{\sigma}(t)$ is adopted, the designed controller contains the item $\frac{\dot{\bar{\sigma}}(t) e(t)}{\bar{\sigma}(t)}$, which could cause the magnitude of control input to be large or even saturation at the initial time.

To better illustrate this issue, consider a simple dynamics system as an illustrative example, i.e.

$$
\dot{x}=u
$$

where $x, u$ are the system state and control input, respectively.

The state tracking error is defined as

$$
e(t)=x-x_{c}
$$

where $x_{c}$ is the continuously differentiable reference command.

From (26) and (27), for FTPPF and PPF, the tracking errors of the transformed system are defined as

$$
\begin{aligned}
& z=\xi-\xi_{c}=\frac{1}{2} \ln \frac{\delta_{\min } \sigma(t)+e(t)}{\delta_{\max } \sigma(t)-e(t)}-\frac{1}{2} \ln \frac{\delta_{\min }}{\delta_{\max }} \\
& =\frac{1}{2} \ln \left(\frac{\delta_{\max }}{\delta_{\min }} \frac{\delta_{\min } \sigma(t)+e(t)}{\delta_{\max } \sigma(t)-e(t)}\right)
\end{aligned}
$$


and

$$
\bar{z}=\frac{1}{2} \ln \left(\frac{\delta_{\max }}{\delta_{\min }} \frac{\delta_{\min } \bar{\sigma}(t)+e(t)}{\delta_{\max } \bar{\sigma}(t)-e(t)}\right)
$$

Then, according to (28), the FTPPC and PPC are designed as

$$
\begin{aligned}
u & =\dot{x}_{c}+\frac{\dot{\sigma}(t) e(t)}{\sigma(t)}-\frac{\eta z}{\mu} \\
& =\dot{x}_{c}+\frac{\left(\sigma_{0}-\sigma_{\infty}\right) e(t)}{T \sigma(t)}\left(\cos \left(\frac{2 \pi t}{T}\right)-1\right)-\frac{\eta z}{\mu}
\end{aligned}
$$

and

$$
\begin{aligned}
& \bar{u}=\dot{x}_{c}+\frac{\dot{\bar{\sigma}}(t) e(t)}{\bar{\sigma}(t)}-\frac{\eta \bar{z}}{\bar{\mu}} \\
& =\dot{x}_{c}-\frac{l\left(\bar{\sigma}_{0}-\bar{\sigma}_{\infty}\right) e^{-l t} e(t)}{\bar{\sigma}(t)}-\frac{\eta \bar{z}}{\bar{\mu}}
\end{aligned}
$$

where $\eta>0$ is design parameter.

From (29), we have $\mu>0, \bar{\mu}>0$. Then, according to the expression of $\bar{z}$ in (36), $-\frac{l\left(\bar{\sigma}_{0}-\bar{\sigma}_{\infty}\right) e^{-l t} e(t)}{\bar{\sigma}(t)}$ and $-\frac{\eta \bar{z}}{\bar{\mu}}$ have the same sign. As $-\frac{\left.l\left(\bar{\sigma}_{0}-\bar{\sigma}_{\infty}\right) e\right\rangle^{l t} e(t)}{\bar{\sigma}(t)}$ and $-\frac{\eta \bar{z}}{\bar{\mu}}$ are added with the same sign, if PPC (38) selects large exponent $l$ in order to quickly reach the final value, $\lim _{t \rightarrow 0^{+}} \frac{\dot{\bar{\sigma}}(t) e(t)}{\bar{\sigma}(t)}=-\frac{l\left(\bar{\sigma}_{0}-\bar{\sigma}_{\infty}\right) e(0)}{\bar{\sigma}_{0}}$ could cause the magnitude of control input to be large or even saturation at the initial time, and may even result in failure to achieve the prescribed performance. For FTPPC (37), we have $\lim _{t \rightarrow 0^{+}} \frac{\dot{\sigma}(t) e(t)}{\sigma(t)}=$ $\lim _{t \rightarrow 0^{+}} \frac{\left(\sigma_{0}-\sigma_{\infty}\right) e(t)}{T \sigma(t)}\left(\cos \left(\frac{2 \pi t}{T}\right)-1\right)=0$. Therefore, the magnitude of control input under FTPPC at the initial time is smaller than PPC.

A set of simulation comparison results between FTPPC and PPC are shown in Figs. $2-3$, where the simulation initial conditions and parameters are selected as $x(0)=$ $0, x_{c}=8, \sigma_{0}=\bar{\sigma}_{0} \Rightarrow 10, \sigma_{\infty} \Rightarrow \bar{\sigma}_{\infty}=0.01, \delta_{\min }=1, \delta_{\max }=1, T=2 s, \eta=1$, and $l=0.8$ for $\mathrm{PPC} 1, l=1.6$ for $\mathrm{PPC} 2, l=2.4$ for PPC 3 , respectively. When the exponent $l$ is selected as $0.8,1.6,2.4$, respectively, the system states under PPC 1-3 converge to final value in $3.9 \mathrm{~s}, 2.7 \mathrm{~s}, 2.1 \mathrm{~s}$, and the magnitude of control inputs under PPC 1-3 at the initial time is 10.3, 16.7, 23.1, respectively. Based on FTPPC, the system state converges to the final value in pre-specified $2 \mathrm{~s}$, and the magnitude of controlinput under FTPPC at the initial time is only 4.0.

Remark 3: For the conventional exponentially decaying PPC, although large exponent $l$ can obtain fast convergence rate, large exponent $l$ also causes the problem of large magnitude of initial control input. According to the task requirements, the FTPPC proposed in this paper can not only preset arbitrarily convergence time without dependence on initial conditions, but also have small magnitude of initial control input. Therefore, FTPPC is more conducive to preassign convergence time and avoid saturation problem. 


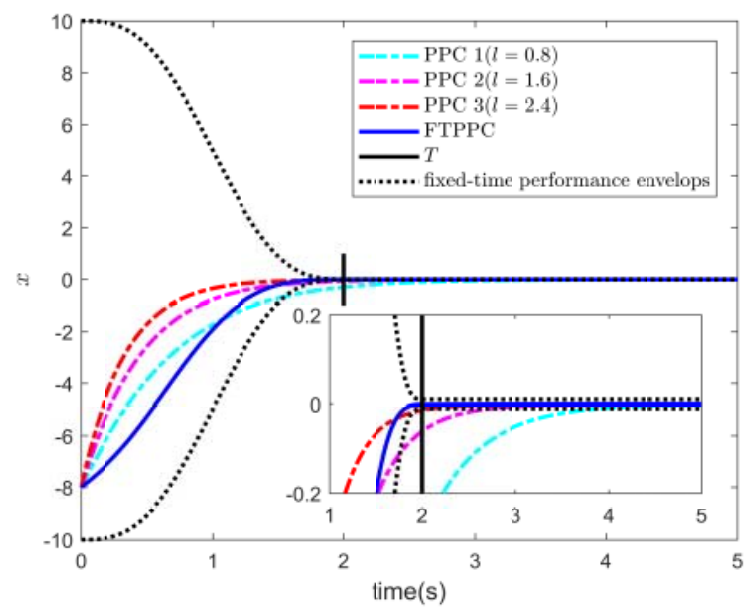

Figure 2. The system state $x$ under PPC and FTPPC.


Figure 3. The control inputs of PPC and FTPPC.

\section{Altitude tracking controller with fixed-time prescribed performance}

\subsection{Error transformed model}

The altitude tracking error $e_{h}(t)=h-h_{c}$ within the constrained fixed-time performance envelops can be formulated by the following inequality

$$
-\delta_{h, \min } \sigma_{h}(t) \leq e_{h}(t) \leq \delta_{h, \max } \sigma_{h}(t)
$$

where $\delta_{h, \min }, \delta_{h, \max }$ are positive design parameters, the fixed-time performance function $\sigma_{h}(t)$ and its parameters $\sigma_{h, 0}, \sigma_{h, \infty}, T_{h}$ are defined the same as (19) and satisfy $-\delta_{h, \min } \sigma_{h}(0) \leq e_{h}(0) \leq \delta_{h, \max } \sigma_{h}(0)$.

By applying the relationship $\sin \gamma \approx \gamma$, and take the derivative of altitude tracking error $e_{h}(t)$, yields

$$
\dot{e}_{h}(t)=V \sin \gamma-\dot{h}_{c} \approx V \gamma-\dot{h}_{c}
$$


By introducing equivalent error transformation $e_{h}(t)=\sigma_{h}(t) s_{h}\left(\xi_{h}(t)\right)$ for the altitude tracking error, it can be obtained

$$
\begin{gathered}
\dot{\xi}_{h}(t)=\mu_{h}\left(\dot{e}_{h}(t)-\frac{\dot{\sigma}_{h}(t) e_{h}(t)}{\sigma_{h}(t)}\right) \\
=\mu_{h}\left(V \gamma-\dot{h}_{c}-\frac{\dot{\sigma}_{h}(t) e_{h}(t)}{\sigma_{h}(t)}\right)
\end{gathered}
$$

where $\sigma_{h}(t), s_{h}\left(\xi_{h}(t)\right), \mu_{h}$ are defined the similar as (19), (25) and (29).

In order to achieve altitude tracking control with fixed-time prescribed performance, the equivalent error transformation need to be incorporated into the original altitude dynamic model (1)-(5). Without loss of generality, the model uncertainties and external disturbances are viewed as lumped disturbances. By replacing the kinematics equation (2) with equivalent error transformation equation (41), let $x_{1}=\xi_{h}(t), x_{2}=\gamma, x_{3}=\alpha, x_{4}=q$, the governing equation for altitude tracking can be equivalently rewritten as the following strict feedback form:

$$
\begin{aligned}
& \dot{x}_{1}=f_{1}+g_{1} x_{2} \\
& \dot{x}_{2}=f_{2}+g_{2} x_{3}+\Delta_{2} \\
& \dot{x}_{3}=f_{3}+g_{3} x_{4} \\
& \dot{x}_{4}=f_{4}+g_{4} \delta_{e}+\Delta_{4}
\end{aligned}
$$

where

$$
\begin{aligned}
& f_{1}=-\mu_{h}\left(\dot{h}_{c}+\frac{\dot{\sigma}_{h}(t) e_{h}(t)}{\sigma_{h}(t)}\right) \\
& g_{1}=\mu_{h} V \\
& f_{2}=\frac{0.5 \rho V^{2} S C_{L 0}}{m V}-\frac{g}{V} \cos \gamma \\
& g_{2}=0.5 \rho V^{2} S C_{L \alpha}+T \\
& f_{3}=-\gamma \\
& g_{3}=1 \\
& f_{4}=0.5 \rho V^{2} S c\left(C_{m \alpha} \alpha+C_{m 0}+\frac{c q}{2 V} C_{m q}\right) / I_{y y} \\
& g_{4}=0.5 \rho V^{2} S c C_{m \delta_{e}} / I_{y y}
\end{aligned}
$$

In addition, $\Delta_{2}, \Delta_{4}$ are the lumped disturbances of the flight-path angle channel and pitch rate channel in the transformed system. Now, the following assumption is introduced.

Assumption 1: The lumped disturbances of the transformed system (42)-(45) are continuously differentiable and the first-order derivatives are bounded, i.e. $\left|\dot{\Delta}_{i}\right| \leq$ $L_{i}, i=2,4$, in which $L_{i}$ are known positive constants. 


\subsection{Robust exact first-order differentiator and finite-time disturbance observer}

Most of the existing literatures on backstepping control select the following three differentiators to obtain differential signals: novel tracking differentiator (TD) proposed in $\mathrm{Bu}$, Wu, Zhang, Ma, and Huang (2015), second-order low-pass differentiator (Kalliny, Elbadawy, \& Elkhamisy, 2018; Pan, Wang, Li, \& Yu, 2018), first-order Levant differentiator (J. Zhang, Xia, Sun, Wang, \& Shen, 2019). The novel tracking differentiator (TD) proposed in Bu et al. (2015) is formulated as

$$
\begin{aligned}
& \dot{\psi}_{1}=\psi_{2} \\
& \dot{\psi}_{2}=-R^{2}\left[a_{1} \sinh \left(l_{1}\left(\psi_{1}-x_{d}\right)\right)+a_{2} \sinh \left(l_{2} \psi_{2} / R\right)\right]
\end{aligned}
$$

where $R>0, a_{1}>0, a_{2}>0, l_{1}>0, l_{2}>0$ are design parameters. $x_{d}$ is the input signal, $\psi_{1}$ and $\psi_{2}$ are the estimations of $x_{d}$ and $\dot{x}_{d}$.

The second-order low-pass differentiator is formulated as

$$
\begin{aligned}
& \dot{\psi}_{1}=\psi_{2} \\
& \dot{\psi}_{2}=-2 \zeta \omega \psi_{2}-\omega^{2}\left(\psi_{1}-x_{d}\right)
\end{aligned}
$$

where $\zeta>0, \omega>0$ are design parameters.

The first-order Levant differentiator is formulated as

$$
\begin{aligned}
& \dot{\psi}_{1}=\iota_{1} \\
& \iota_{1}=-k_{1}\left|\psi_{1}-x_{d}\right|^{\frac{1}{2}} \operatorname{sign}\left(\psi_{1}-x_{d}\right)+\psi_{2} \\
& \dot{\psi}_{2}=-k_{2} \operatorname{sign}\left(\psi_{2}-\iota_{1}\right)
\end{aligned}
$$

where $k_{1}>0, k_{2}>0$ are design parameters.

In this paper, the robust exact first-order differentiator (12) proposed in Angulo et al. (2013) is used to obtain the differential signal in finite-time. A simulation comparison research is conducted to test the superiority of the robust exact first-order differentiator compared with the above three differentiators. The simulation parameters are shown in Table 1 . The input command signal $x_{d}$ is selected as the following function appended with a random noise of variance 0.05 .

$$
x_{d}= \begin{cases}0, & 0 \leq t<0.1 \\ 1+1.2 \sin (0.5 t)+1.6 \cos (0.4 t), & t \geq 0.1\end{cases}
$$

Table 1. The simulation parameters of the four differentiators.

Differentiator The values of design parameters

Tracking Differentiator (47) R=10, $a_{1}=5, a_{2}=4, l_{1}=1, l_{2}=1$

Second-order low-pass differentiator (48) $\quad \zeta=0.707, \omega=15$

First-order Levant differentiator (49) $\quad k_{1}=10, k_{2}=12$

Robust exact first-order differentiator (12) $\quad k_{1}=10, k_{2}=12, k_{3}=20, k_{4}=20, \tau=0.1, T_{\vartheta}=0.3$

The simulation results of the four differentiators are shown in Figs. 4-5. As shown in Fig. 4, by selecting appropriate parameters, each differentiator can track the command signal in a short time. It can be seen from Fig. 5 that the four differentiators, 
especially the tracking differentiator, obtain smooth differential signals in a short time. In the step phase where the input command signal changes rapidly, the maximum differential tracking errors of the differentiators (47)-(49) are 26.2, 17.8, 2.5, respectively, while the robust exact first-order differentiator is only 1.9, the maximum differential tracking error of the robust exact first-order differentiator is smaller than the other three differentiators. As can be seen from Fig. 4, the convergence rate of the robust exact first-order differentiator is faster than first-order Levant differentiator. Therefore, based on the robust exact first-order differentiator, the controller can obtain high-quality command tracking signals and differential signals in finite-time.
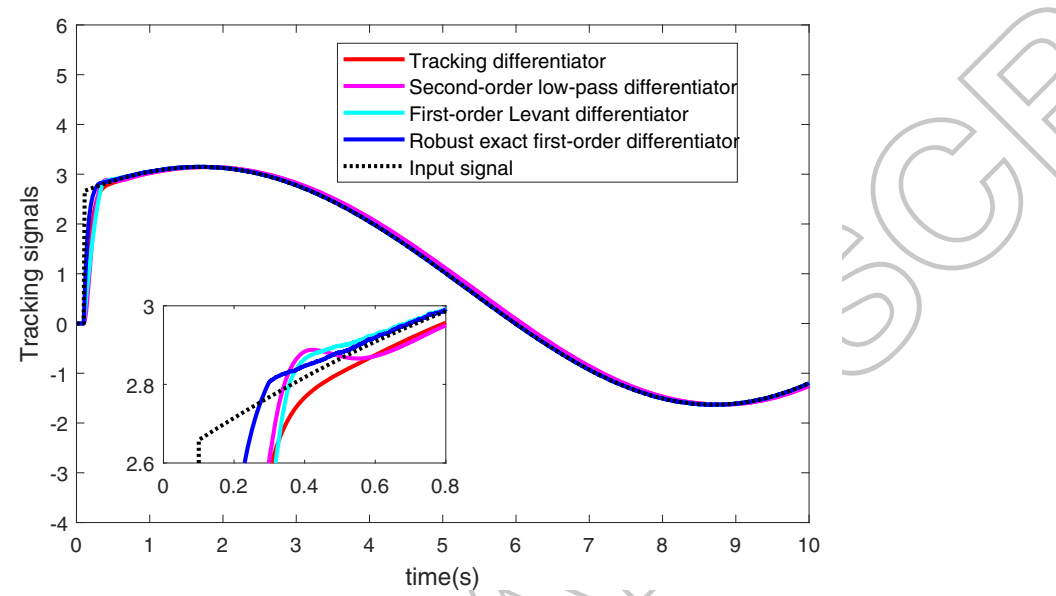

Figure 4. Tracking signals of the four differentiators.

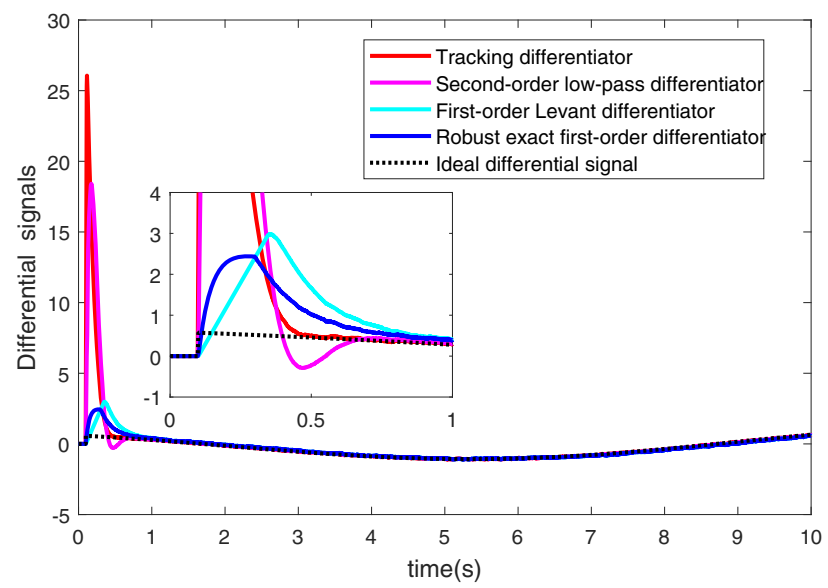

Figure 5. Differential signals of the four differentiators.

Moreover, based on the robust exact first-order differentiator, finite-time disturbance observers are designed to accurately estimate and compensate lumped disturbances in transformed system (42)-(45).

Theorem 1: For the transformed system (42)-(45) with lumped disturbances under 
Assumption 1, construct the finite-time disturbance observers as follows

$$
\begin{aligned}
& \dot{\hat{x}}_{\mathrm{i}}=f_{i}+g_{i} u_{i}-r_{i, 1} \vartheta \operatorname{sig}\left(\hat{x}_{\mathrm{i}}-x_{\mathrm{i}}\right)^{1 / 2}-r_{i, 3}(1-\vartheta) \operatorname{sig}\left(\hat{x}_{\mathrm{i}}-x_{\mathrm{i}}\right)^{(2+\tau) / 2}+\hat{\Delta}_{i} \\
& \dot{\hat{\Delta}}_{i}=-r_{i, 2} \vartheta \operatorname{sign}\left(\hat{x}_{\mathrm{i}}-x_{\mathrm{i}}\right)-r_{i, 4}(1-\vartheta) \operatorname{sig}\left(\hat{x}_{\mathrm{i}}-x_{\mathrm{i}}\right)^{1+\tau}
\end{aligned}
$$

where $i=2,4, u_{2}=x_{3}, u_{4}=\delta_{e} . \hat{x}_{i}$ and $\hat{\Delta}_{i}$ are the estimates of $x_{i}$ and $\Delta_{i}$, respectively. $r_{i, 1}, r_{i, 2}$ are positive design parameters which satisfy $r_{i, 1} \geq 1.5 \sqrt{L_{i}}, r_{i, 2} \geq 1.1 L_{i}$ with $L_{i}$ defined in Assumption 1. $r_{i, 3}, r_{i, 4}$ are selected as $r_{i, 3}>0, r_{i, 4}>0$ and $\tau$ is chosen small enough. The function $\vartheta:[0, \infty) \rightarrow\{0,1\}$ is defined by $\vartheta=0$ if $t \leq T_{\vartheta}$ and otherwise $\vartheta=1$ with an arbitrarily positive constant $T_{\vartheta}>0$. The stability of the disturbance observer can be achieved in finite-time $T_{i, 1}$ such that $\hat{\Delta}_{i} \rightarrow \Delta_{i}$ for $t<T_{i, 1}$ and $\hat{\Delta}_{i}=\Delta_{i}$ for $t \geq T_{i, 1}$.

Proof: Subtracting the disturbance observers (51) from the transformed system (43) and (45), it can be obtained

$$
\begin{aligned}
& \dot{\tilde{x}}_{\mathrm{i}}=-r_{i, 1} \vartheta \operatorname{sig}\left(\tilde{x}_{\mathrm{i}}\right)^{1 / 2}-r_{i, 3}(1-\vartheta) \operatorname{sig}\left(\tilde{x}_{\mathrm{i}}\right)^{(2+\tau) / 2} \\
& \dot{\tilde{\Delta}}_{i}=-r_{i, 2} \vartheta \operatorname{sign}\left(\tilde{x}_{\mathrm{i}}\right)-r_{i, 4}(1-\vartheta) \operatorname{sig}\left(\tilde{x}_{\mathrm{i}}\right)^{1+\tau}-\Delta_{i}
\end{aligned}
$$

where $i=2,4, \tilde{x}_{i}=\hat{x}_{i}-x_{i}, \tilde{\Delta}_{i}=\hat{\Delta}_{i}-\Delta_{i}$. With the parameters being selected in Theorem 1, the stability and finite-time convergence property of (52) have been proved in Angulo et al. (2013). Therefore, the disturbance estimate errors $\tilde{\Delta}_{i}$ converge to 0 in finite-time $T_{i, 1}$, such that $\hat{\Delta}_{i} \rightarrow \Delta_{i}$ for $t<T_{i, 1}$ and $\hat{\Delta}_{i}=\Delta_{i}$ for $t \geq T_{i, 1}$.

\subsection{Backstepping controller}

The aim of backstepping controller is to generate the appropriate elevator deflection $\delta_{e}$ which guarantees that the altitude tracking equivalent error $x_{1}$ converges to the specified small neighborhood of the desired reference signal in spite of lumped disturbances. The tracking errors of the transformed system (42)-(45) are defined as

$$
\begin{aligned}
& z_{1}=x_{1}-x_{1, d} \\
& z_{2}=x_{2}-x_{2, c} \\
& z_{3}=x_{3}-x_{3, c} \\
& z_{4}=x_{4}-x_{4, c}
\end{aligned}
$$

where $x_{1, d}=0.5 \ln \left(\delta_{h, \min } / \delta_{h, \max }\right)$ is the desired reference signal of transformed error $x_{1}=\xi_{h}(t) \cdot x_{2, c}, x_{3, c}, x_{4, c}$ are the outputs of robust exact first-order differentiators with the yirtual control signals $x_{2, d}, x_{3, d}, x_{4, d}$ as the inputs. The finite-time robust exact first-order differentiators are designed as follows

$$
\begin{aligned}
& \dot{\psi}_{i, 1}=-\kappa_{i, 1} \vartheta_{0} \operatorname{sig}\left(\psi_{i, 1}-x_{i, d}\right)^{1 / 2}-\kappa_{i, 3}\left(1-\vartheta_{0}\right) \operatorname{sig}\left(\psi_{i, 1}-x_{i, d}\right)^{\left(2+\tau_{0}\right) / 2}+\psi_{i, 2} \\
& \dot{\psi}_{i, 2}=-\kappa_{i, 2} \vartheta_{0} \operatorname{sign}\left(\psi_{i, 1}-x_{i, d}\right)-\kappa_{i, 4}\left(1-\vartheta_{0}\right) \operatorname{sig}\left(\psi_{i, 1}-x_{i, d}\right)^{1+\tau_{0}}
\end{aligned}
$$

where $x_{i, c}=\psi_{i, 1}, \dot{x}_{i, c}=\psi_{i, 2}, i=2,3,4$. The positive design parameters $\kappa_{i, 1}, \kappa_{i, 2}$ are selected based on the original first-order sliding mode differentiator (Levant, 2003); $\kappa_{i, 3}, \kappa_{i, 4}$ are selected as $\kappa_{i, 3}>0, \kappa_{i, 4}>0$ and $\tau_{0}$ is chosen small enough. The function $\vartheta_{0}:[0, \infty) \rightarrow\{0,1\}$ is defined by $\vartheta_{0}=0$ if $t \leq T_{\vartheta_{0}}$ and otherwise $\vartheta_{0}=1$ with an 
arbitrarily positive constant $T_{\vartheta_{0}}>0$. According to Lemma 1, robust exact first-order differentiator is finite-time exact.

Choose the virtual control signals $x_{i, d}, i=2,3,4$ and the control input $\delta_{e}$ as follows

$$
\begin{aligned}
& x_{2, d}=\frac{1}{g_{1}}\left[-f_{1}-\eta_{1} z_{1}+\dot{x}_{1, d}-\beta_{1} \operatorname{sig}\left(\varepsilon_{1}\right)^{a}-\lambda_{1} \operatorname{sig}\left(\zeta_{1}\right)^{b}\right] \\
& x_{3, d}=\frac{1}{g_{2}}\left[-f_{2}-\eta_{2} z_{2}+\dot{x}_{2, c}-\hat{\Delta}_{2}-g_{1} z_{1}-\beta_{2} \operatorname{sig}\left(\varepsilon_{2}\right)^{a}-\lambda_{2} \operatorname{sig}\left(\zeta_{2}\right)^{b}\right] \\
& x_{4, d}=\frac{1}{g_{3}}\left[-f_{3}-\eta_{3} z_{3}+\dot{x}_{3, c}-g_{2} z_{2}-\beta_{3} \operatorname{sig}\left(\varepsilon_{3}\right)^{a}-\lambda_{3} \operatorname{sig}\left(\zeta_{3}\right)^{b}\right] \\
& \delta_{e}=\frac{1}{g_{4}}\left[-f_{4}-\eta_{4} z_{4}+\dot{x}_{4, c}-\hat{\Delta}_{4}-g_{3} z_{3}-\beta_{4} \operatorname{sig}\left(\varepsilon_{4}\right)^{a}-\lambda_{4} \operatorname{sig}\left(\zeta_{4}\right)^{b}\right]
\end{aligned}
$$

where $\eta_{i}>0, \beta_{i}>0, \lambda_{i}>0, i=1,2,3,4$ are positive design parameters to be determined. $a, b$ are positive constants and be set as $0<a<1,0<b<1, \zeta_{i}$ are the compensated tracking error signals which have the following definition

$$
\zeta_{i}=z_{i}-\varepsilon_{i}, \quad i=1,2,3,4
$$

The error compensation signals $\varepsilon_{i}, i=1,2,3,4$ are defined as follows

$$
\begin{aligned}
& \dot{\varepsilon}_{1}=-\eta_{1} \varepsilon_{1}+g_{1}\left(x_{2, c}-x_{2, d}\right)+g_{1} \varepsilon_{2}-\beta_{1} \operatorname{sig}\left(\varepsilon_{1}\right)^{a} \\
& \dot{\varepsilon}_{2}=-\eta_{2} \varepsilon_{2}+g_{2}\left(x_{3, c}-x_{3, d}\right)-g_{1} \varepsilon_{1}+g_{2} \varepsilon_{3}-\beta_{2} \operatorname{sig}\left(\varepsilon_{2}\right)^{a} \\
& \dot{\varepsilon}_{3}=-\eta_{3} \varepsilon_{3}+g_{3}\left(x_{4, c}-x_{4, d}\right)-g_{2} \varepsilon_{2}+g_{3} \varepsilon_{4}-\beta_{3} \operatorname{sig}\left(\varepsilon_{3}\right)^{a} \\
& \dot{\varepsilon}_{4}=-\eta_{4} \varepsilon_{4}-g_{3} \varepsilon_{3}-\beta_{4} \operatorname{sig}\left(\varepsilon_{4}\right)^{a}
\end{aligned}
$$

where $\varepsilon_{i}(0)=0, i=1,2,3,4$.

Theorem 2: Consider the transformed error system (42)-(45) with the lumped disturbances under Assumption 1, the finite-time disturbance observers (51) are used to accurately estimate and compensate lumped disturbances, the robust exact firstorder differentiators are chosen as in (57), an improved finite-time error compensation mechanism is designed as in (63)-(66), then we can select the virtual control signals (58)-(60) and the control law (61), such that the transformed error converges to the specified small neighborhood of desired reference signal in finite-time, which guarantees the transient and steady-state performance of altitude tracking error within constrained fixed-time performance envelops.

Proof. The complete proof procedure of Theorem 2 is divided into the following five steps.

Step 1: Choose the Lyapunov function candidate $V_{1}$ as

$$
V_{1}=\frac{1}{2} \zeta_{1}^{2}
$$

Taking the time derivative of $V_{1}$, yields

$$
\begin{aligned}
& \dot{V}_{1}=\zeta_{1} \dot{\zeta}_{1}=\zeta_{1}\left(\dot{x}_{1}-\dot{x}_{1, d}-\dot{\varepsilon}_{1}\right) \\
& =\zeta_{1}\left[f_{1}+g_{1}\left(x_{2, c}-x_{2, d}\right)+g_{1}\left(z_{2}+x_{2, d}\right)-\dot{x}_{1, d}-\dot{\varepsilon}_{1}\right]
\end{aligned}
$$

Substituting virtual control signal $x_{2, d}$ and the derivative of error compensation 
signal $\varepsilon_{1}$, it can be obtained

$$
\begin{aligned}
& \dot{V}_{1}=\zeta_{1}\left[g_{1}\left(z_{2}-\varepsilon_{2}\right)+\eta_{1}\left(\varepsilon_{1}-z_{1}\right)-\lambda_{1} \operatorname{sig}\left(\zeta_{1}\right)^{b}\right] \\
& =\zeta_{1}\left[-\eta_{1} \zeta_{1}+g_{1} \zeta_{2}-\lambda_{1} \operatorname{sig}\left(\zeta_{1}\right)^{b}\right] \\
& =-\eta_{1} \zeta_{1}^{2}+g_{1} \zeta_{1} \zeta_{2}-\lambda_{1}\left|\zeta_{1}\right|^{b+1}
\end{aligned}
$$

Step 2: Choose the Lyapunov function candidate $V_{2}$ as

$$
V_{2}=V_{1}+\frac{1}{2} \zeta_{2}^{2}
$$

Taking the time derivative of $V_{2}$, yields

$$
\begin{aligned}
& \dot{V}_{2}=\dot{V}_{1}+\zeta_{2} \dot{\zeta}_{2}=\dot{V}_{1}+\zeta_{2}\left(\dot{x}_{2}-\dot{x}_{2, c}-\dot{\varepsilon}_{2}\right) \\
& =\dot{V}_{1}+\zeta_{2}\left[f_{2}+g_{2}\left(x_{3, c}-x_{3, d}\right)+g_{2}\left(z_{3}+x_{3, d}\right)+\Delta_{2}-\dot{x}_{2, c}-\dot{\varepsilon}_{2}\right]
\end{aligned}
$$

Substituting virtual control signal $x_{3, d}$ and the derivative of error compensation signal $\varepsilon_{2}$, it can be obtained

$$
\dot{V}_{2}=-\eta_{1} \zeta_{1}^{2}-\eta_{2} \zeta_{2}^{2}-\lambda_{1}\left|\zeta_{1}\right|^{b+1}-\lambda_{2}\left|\zeta_{2}\right|^{b+1}+g_{2} \zeta_{2} \zeta_{3}-\zeta_{2} \tilde{\Delta}_{2}
$$

Step 3: Choose the Lyapunov function candidate $\sqrt{3}$ as

$$
V_{3}=\widehat{V}_{2}+\frac{1}{2} \zeta_{3}^{2}
$$

Taking the time derivative of $V_{3}$, yields

$$
\begin{aligned}
& \dot{V}_{3}=\dot{V}_{2}+\zeta_{3} \dot{\zeta}_{3}=\dot{V}_{2}+\zeta_{3}\left(\dot{x}_{3}-\dot{x}_{3, c}-\dot{\varepsilon}_{3}\right) \\
& =\dot{V}_{2}+\zeta_{3}\left[f_{3}+g_{3}\left(x_{4, c}-x_{4, d}\right)+g_{3}\left(z_{4}+x_{4, d}\right)-\dot{x}_{3, c}-\dot{\varepsilon}_{3}\right]
\end{aligned}
$$

Substituting virtual control signal $x_{4, d}$ and the derivative of error compensation signal $\varepsilon_{3}$, it can be obtained

$$
\dot{V}_{3}=-\sum_{i=1}^{3} \eta_{i} \zeta_{i}^{2}-\sum_{i=1}^{3} \lambda_{i}\left|\zeta_{i}\right|^{b+1}+g_{3} \zeta_{3} \zeta_{4}-\zeta_{2} \tilde{\Delta}_{2}
$$

Step 4: Choose the Lyapunov function candidate $V_{4}$ as

$$
V_{4}=V_{3}+\frac{1}{2} \zeta_{4}^{2}
$$

Taking the time derivative of $V_{4}$, yields

$$
\begin{gathered}
\dot{V}_{4}=\dot{V}_{3}+\zeta_{4} \dot{\zeta}_{4}=\dot{V}_{3}+\zeta_{4}\left(\dot{x}_{4}-\dot{x}_{4, c}-\dot{\varepsilon}_{4}\right) \\
=\dot{V}_{3}+\zeta_{4}\left[f_{4}+g_{4} \delta_{e}+\Delta_{4}-\dot{x}_{4, c}-\dot{\varepsilon}_{4}\right]
\end{gathered}
$$


Substituting the control law $\delta_{e}$ and the derivative of error compensation signal $\varepsilon_{4}$, it can be obtained

$$
\dot{V}_{4}=-\sum_{i=1}^{4} \eta_{i} \zeta_{i}^{2}-\sum_{i=1}^{4} \lambda_{i}\left|\zeta_{i}\right|^{b+1}-\zeta_{2} \tilde{\Delta}_{2}-\zeta_{4} \tilde{\Delta}_{4}
$$

Since the disturbance estimate errors $\tilde{\Delta}_{i}=\hat{\Delta}_{i}-\Delta_{i}, i=2,4$, converge to 0 in finite-time $T_{2,1}, T_{4,1}$, respectively. When $t=T_{1} \geq \max \left\{\mathrm{T}_{2,1}, \mathrm{~T}_{4,1}\right\}$, there are $\tilde{\Delta}_{2}=$ $0, \tilde{\Delta}_{4}=0$. According to Lemma 3 , (78) becomes

$$
\dot{V}_{4} \leq-\eta V_{4}-\lambda V_{4}^{(1+b) / 2}
$$

where $\eta=2 \min \left(\eta_{i}\right), \lambda=2^{(1+b) / 2} \min \left(\lambda_{i}\right), i=1,2,3,4$. According to Lemma 4, $\zeta_{i}, i=1,2,3,4$ converge to 0 in finite-time $T_{2}$, and the expression of $T_{2}$ is as follows:

$$
T_{2} \leq \frac{2}{(1-b) \eta} \ln \left(\frac{\eta V_{4}(0)^{(1-b) / 2}+\lambda}{\lambda}\right)
$$

Since $z_{i}=\zeta_{i}+\varepsilon_{i}$, the convergence property of $\varepsilon_{i}, i \bumpeq 1,2,3,4$ are analyzed next.

Step 5: Choose the Lyapunov function of the error compensation system as follows

$$
V_{\varepsilon}=\frac{1}{2} \sum_{i=1}^{4} \varepsilon_{i}^{2}
$$

Taking the time derivative of $V_{\varepsilon}$, yields

$$
\begin{aligned}
& \dot{V}_{\varepsilon}=-\sum_{i=1}^{4} \eta_{i} \varepsilon_{i}^{2}-\sum_{i=1}^{4} \beta_{i}\left|\varepsilon_{i}\right|^{a+1}+\sum_{i=1}^{3}\left(x_{i+1, c}-x_{i+1, d}\right) g_{i} \varepsilon_{i} \\
& \leq-\eta V_{\varepsilon}-\beta Y_{\varepsilon}^{(1+a) / 2}+\sum_{i=1}^{3}\left(x_{i+1, c}-x_{i+1, d}\right) g_{i} \varepsilon_{i}
\end{aligned}
$$

where $\eta=2 \min \left(\eta_{i}\right), \beta=2^{(1+a) / 2} \min \left(\beta_{i}\right), i=1,2,3,4$. According to Lemma 2 , if there are noises in the virtual control signals, $\left|x_{i+1, c}-x_{i+1, d}\right| \leq \nu_{i+1}, i=1,2,3$ can be obtained in finite-time $T_{i, 3}, i=1,2,3$. From (46), we have $\left|g_{i}\right| \leq g_{0}$, where $g_{0}$ is a known positive constant. When $t \geq T_{3}=\max \left\{T_{i, 3}\right\}, i=1,2,3$, we have

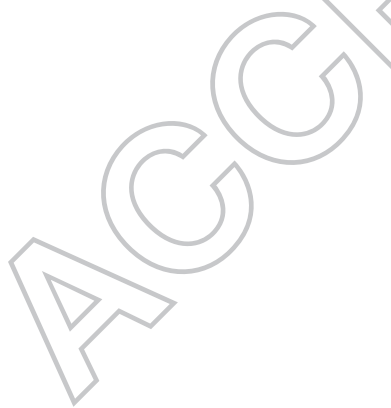

$$
\begin{aligned}
& \sum_{i=1}^{3}\left(x_{i+1, c}-x_{i+1, d}\right) g_{i} \varepsilon_{i} \leq \sum_{i=1}^{3} \nu_{i+1} g_{0}\left|\varepsilon_{i}\right| \\
& \leq \sum_{i=1}^{3}\left(\frac{l_{0} \varepsilon_{i}^{2}}{2}+\frac{\nu_{i+1}^{2} g_{0}^{2}}{2 l_{0}}\right) \\
& \leq l_{0} V_{\varepsilon}+\sum_{i=1}^{3} \frac{\nu_{i+1}^{2} g_{0}^{2}}{2 l_{0}}
\end{aligned}
$$

where $l_{0}>0$ is the selected positive constant, substituting (83) into (82), it can be 
obtained

$$
\dot{V}_{\varepsilon} \leq-\eta_{0} V_{\varepsilon}-\beta V_{\varepsilon}^{(1+a) / 2}+\chi
$$

where $\eta_{0}=\eta-l_{0}>0, \chi=\sum_{i=1}^{3} \frac{\nu_{i+1}^{2} g_{0}^{2}}{2 l_{0}}$.

The inequality (84) can be written as

$$
\dot{V}_{\varepsilon} \leq-(1-p) \eta_{0} V_{\varepsilon}-p \eta_{0} V_{\varepsilon}-\beta V_{\varepsilon}^{(1+a) / 2}+\chi
$$

or

$$
\dot{V}_{\varepsilon} \leq-\eta_{0} V_{\varepsilon}-(1-p) \beta V_{\varepsilon}^{(1+a) / 2}-p \beta V_{\varepsilon}^{(1+a) / 2}+\chi
$$

where $0<p<1$ is the designed positive constant.

From (85), if $V_{\varepsilon} \geq \frac{\chi}{p \eta_{0}}$, we have

$$
\dot{V}_{\varepsilon} \leq-(1-p) \eta_{0} V_{\varepsilon}-\beta V_{\varepsilon}^{(1+a) / 2}
$$

Then, by Lemma $4, V_{\varepsilon}$ converges into the following region in finite-time $T_{1,4}$.

$$
V_{\varepsilon} \leq \frac{\chi}{p \eta_{0}}
$$

The corresponding time $T_{1,4}$ can be given as

$$
T_{1,4} \leq \frac{2}{(1-p)(1-a) \eta_{0}} \ln \frac{(1-p) \eta_{0} V(0)_{\varepsilon}^{(1-a) / 2}+\beta}{\beta}
$$

From (86), if $V_{\varepsilon} \geq\left(\frac{x}{p B}\right)^{2 /(1+a)}$, we have

$$
\dot{V}_{\varepsilon} \leq-\eta_{0} V_{\varepsilon}-(1-p) \beta V_{\varepsilon}^{(1+a) / 2}
$$

Then, by Lemma $4, V_{\varepsilon}$ converges into the following region in finite-time $T_{2,4}$.

$$
V_{\varepsilon} \leq\left(\frac{\chi}{p \beta}\right)^{2 /(1+a)}
$$

The corresponding time $T_{2,4}$ can be given as

$$
T_{2,4} \leq \frac{2}{\eta_{0}(1-a)} \ln \frac{\eta_{0} V(0)_{\varepsilon}^{(1-a) / 2}+(1-p) \beta}{(1-p) \beta}
$$

Combining together (88) and (91), we can obtain

$$
V_{\varepsilon} \leq \min \left\{\frac{\chi}{p \eta_{0}},\left(\frac{\chi}{p \beta}\right)^{2 /(1+a)}\right\}
$$


Since $\varepsilon_{i}^{2} \leq 2 V_{\varepsilon}, i=1,2,3,4$, from (93), $\varepsilon_{i}$ finally converge into region

$$
\left|\varepsilon_{i}\right| \leq \min \left\{\left(\frac{2 \chi}{p \eta_{0}}\right)^{1 / 2}, \sqrt{2}\left(\frac{\chi}{p \beta}\right)^{1 /(1+a)}\right\}
$$

From (89) and (92), the convergence time of $T_{4}$ can be estimated as $T_{4} \leq$ $\max \left\{T_{1,4}, T_{2,4}\right\}$.

According to the analysis of step $1 \sim 4$, we have $\left|\zeta_{i}\right|=0$. Since $z_{i}=\zeta_{i}+\varepsilon_{i}, i=$ $1,2,3,4$, for $t \geq T_{1}+T_{2}+T_{3}+T_{4}$, then it follows

$$
\begin{aligned}
& \left|z_{1}\right|=\left|\xi_{h}-x_{1, d}\right| \leq\left|\zeta_{1}\right|+\left|\varepsilon_{1}\right| \\
& \leq \min \left\{\left(\frac{2 \chi}{p \eta_{0}}\right)^{1 / 2}, \sqrt{2}\left(\frac{\chi}{p \beta}\right)^{1 /(1+a)}\right\}
\end{aligned}
$$

It can be shown that the signals $z_{i}, \zeta_{i}, \varepsilon_{i}, i=1,2,3,4$ are bounded, From (95), the transformed error $\xi_{h}(t)$ converges to the specified small neighborhood of the reference signal in finite-time in spite of lumped disturbances, which guarantees the transient and steady-state performance of altitude tracking error within constrained fixed-time performance envelops.

This completes the proof.

Remark 4: In the proof process of step 5, it is assumed that the virtual control signals have the influence of noises, so that $\left|x_{i+1, c}-x_{i+1, d}\right| \leq \nu_{i+1}, i=1,2,3$. Note that if the virtual control signals are not influence by noises, then $x_{i+1, c}-x_{i+1, d}=0, i=1,2,3$ can be realized in finite-time, that is $\nu_{i+1} \neq 0, i=1,2,3$. Then $z_{i}=\zeta_{i}+\varepsilon_{i}, i=1,2,3,4$ converge to 0 in finite-time.

Remark 5: The adaptive neural network prescribed performance control (Guo, Zhang, Celler, \& Su, 2019) and adaptiye fuzzy prescribed performance control (Li \& Tong, 2018) eliminate the influence of uncertain nonlinearities by employing neuro/fuzzy approximating structures. However, as extra adaptive parameters have to be updated and cumbersome parameters have to be introduced, the introduction of neuro/fuzzy approximating structures increase the complexity and extra calculations. In this paper, the disturbance observers are used to estimate and compensate the lumped disturbances, avoiding the introduction of complex neuro/fuzzy approximating structures. In addition, the use of robust exact first-order differentiator at each step of the design progress in this paper avoids the repeated differentiations of the virtual signals and eliminates "explosion of complexity" phenomenon of the conventional backstepping control.

Remark 6: It is worth mentioning the pioneering work on fixed-time control in Polyakov (2012), in which stabilizing polynomial feedbacks algorithm and special modification of terminal second order sliding mode control algorithm provide fixed-time stability of the closed-loop system and allow to adjust a guaranteed settling time independent of initial conditions. In this paper, a novel fixed-time prescribed performance controller is used to achieve fixed-time control. The novel fixed-time performance function is developed to impose priori performance envelops on tracking error. The key idea is to use error transformed technology that the constrained fixed-time performance envelops are changed into unconstrained equivalent error. Then, the equivalent error system is stabilized based on backstepping approach, which ensures the transient and steady-state performance of tracking error within constrained fixed-time performance envelops. 


\section{Airspeed tracking controller with fixed-time prescribed performance}

According to (1), the airspeed subsystem of the UAV longitudinal model can be written as the following form

$$
\dot{V}=f_{V}+g_{V} \delta_{T}+\Delta_{V}
$$

where

$$
\begin{aligned}
& f_{V}=-g \sin \gamma-0.5 \rho V^{2} S C_{D} / m \\
& g_{V}=T_{\max } \cos \alpha / m
\end{aligned}
$$

In addition, $\Delta_{V}$ is the lumped disturbances including model uncertainties and external disturbances. Now, the following assumption is introduced.

Assumption 2: The lumped disturbances of the airspeed subsystem (1) is continuously differentiable and the first-order derivative is bounded, i.e. $\left|\Delta_{V}\right| \leq_{V}$, in which $L_{V}$ is a known positive constant.

Based on the robust exact first-order differentiator, finite-time disturbance observer is designed to accurately estimate and compensate lumped disturbances in airspeed subsystem (1) as follows

$$
\begin{aligned}
& \dot{\hat{V}}=f_{V}+g_{V} \delta_{T}-r_{V, 1} \vartheta \operatorname{sig}(\hat{V}-V)^{1 / 2}-r_{V, 3}(1-\vartheta) \operatorname{sig}\left(\hat{V}^{2}-V\right)^{(2+\tau) / 2}+\hat{\Delta}_{V} \\
& \dot{\hat{\Delta}}_{V}=-r_{V, 2} \vartheta \operatorname{sign}(\hat{V}-V)-r_{V, 4}(1-\vartheta) \operatorname{sig}(\hat{V}-V)^{1+\tau}
\end{aligned}
$$

where $\hat{V}$ and $\hat{\Delta}_{V}$ are the estimates of $K$ and $\Delta_{V}$, respectively. Besides, the parameters $r_{V, 1}, r_{V, 2}, r_{V, 3}, r_{V, 4}, \vartheta, \tau$ are defined the similar as disturbance observers (51).

Then, by the similar analysis as Theorem 1, the stability of the disturbance observer can be achieved in finite-time $T_{V, 1}$ such that $\hat{\Delta}_{V} \rightarrow \Delta_{V}$ for $t<T_{V, 1}$ and $\hat{\Delta}_{V}=\Delta_{V}$ for $t \geq T_{V, 1}$.

The airspeed tracking error $e_{V}(t)=V-V_{c}$ within the constrained fixed-time performance envelops can be formulated by the following inequality

$$
-\delta_{V, \min } \sigma_{V}(t) \leq e_{V}(t) \leq \delta_{V, \max } \sigma_{V}(t)
$$

where $\delta_{V \text {,min }}, \delta_{V, \text { max }}$ are positive design parameters, the fixed-time performance function $\sigma_{y}(t)$ and its parameters $\sigma_{V, 0}, \sigma_{V, \infty}, T_{V}$ are defined the similar as (19) and satisfy $-\delta_{V, \min \sigma_{V}(0)} \leq e_{V}(0) \leq \delta_{V, \max } \sigma_{V}(0)$.

By introducing an equivalent error transformation $e_{V}(t)=\sigma_{V}(t) s_{V}\left(\xi_{V}(t)\right)$ for the airspeed tracking error, it can be obtained

$$
\dot{\xi}_{V}(t)=\mu_{V}\left[\dot{e}_{V}(t)-\frac{\dot{\sigma}_{V}(t) e_{V}(t)}{\sigma_{V}(t)}\right]
$$

where $\sigma_{V}(t), s_{V}\left(\xi_{V}(t)\right), \mu_{V}$ are defined the similar as (19), (25) and (29).

Take the derivative of airspeed tracking error $e_{V}(t)$, yields

$$
\dot{e}_{V}(t)=f_{V}+g_{V} \delta_{T}+\Delta_{V}-\dot{V}_{c}
$$


Substituting (101) into (100), it can be obtained

$$
\dot{\xi}_{V}(t)=\mu_{V}\left[f_{V}+g_{V} \delta_{T}+\Delta_{V}-\dot{V}_{c}-\frac{\dot{\sigma}_{V}(t) e_{V}(t)}{\sigma_{V}(t)}\right]
$$

By the similar formula as (27), the desired reference signal of transformed error $\xi_{V}(t)$ is

$$
\xi_{V, c}=\frac{1}{2} \ln \frac{\delta_{V, \min }}{\delta_{V, \max }}
$$

Define the error signal

$$
z_{V}=\xi_{V}(t)-\xi_{V, c}
$$

Based on the finite-time disturbance observer (98), the airspeed control law is designed as

$$
\delta_{T}=\frac{1}{g_{V}}\left[-f_{V}-\hat{\Delta}_{V}+\dot{V}_{c}+\frac{\dot{\sigma}_{V}(t) e_{V}(t)}{\sigma_{V}(t)}-\frac{\eta_{V, 1} z_{V}}{\mu_{V}}-\frac{\eta_{V, 2}}{\mu_{V}} \operatorname{sig}\left(z_{V}\right)^{b_{V}}\right]
$$

Theorem 3: Consider the transformed error system (102) with the lumped disturbances under Assumption 2, the finite-time disturbance observer (98) is used to accurately estimate and compensate lumped disturbances, then we can select the control law (105), such that the transformed error converges to the reference signal in finite-time, which guarantees the transient and steady-state performance of airspeed tracking error within constrained fixed-time performance envelops.

Proof: A Lyapunov function is designed as

$$
V_{V}=\frac{1}{2} z_{V}^{2}
$$

Take the time derivative of (106) yields

$$
\begin{aligned}
& \dot{V}_{V}=z_{V} \dot{z}_{V} \\
& =z_{V} \mu_{V}\left[f_{V}+g_{V} \delta_{T}+\Delta_{V}-\dot{V}_{c}-\frac{\dot{\sigma}_{V}(t) e_{V}(t)}{\sigma_{V}(t)}\right] \\
& =-\eta_{V, 1} z_{V}^{2}-\eta_{V, 2}\left|z_{V}\right|^{b_{V}+1}-z_{V} \mu_{V} \tilde{\Delta}_{V} \\
& =-\varpi_{1} V_{V}-\varpi_{2} V_{V}^{\left(b_{V}+1\right) / 2}-z_{V} \mu_{V} \tilde{\Delta}_{V}
\end{aligned}
$$

where $\varpi_{1}=2 \eta_{V, 1}, \varpi_{2}=2^{\left(b_{V}+1\right) / 2} \eta_{V, 2}$. Since the disturbance estimate error $\tilde{\Delta}_{V}=$ $\hat{\Delta}_{V}-\Delta_{V}$ converges to 0 in finite-time $T_{V, 1}$, when $t \geq \mathrm{T}_{V, 1}$, there is $\tilde{\Delta}_{V}=0$, and (107) becomes

$$
\dot{V}_{V}=-\varpi_{1} V_{V}-\varpi_{2} V_{V}^{\left(b_{V}+1\right) / 2}
$$

According to Lemma $4, z_{V}$ converges to 0 in finite-time $T_{V, 2}$, and the convergence 
time of $T_{V, 2}$ can be estimated as follows

$$
T_{V, 2} \leq \frac{2}{\left(1-b_{V}\right) \varpi_{1}} \ln \left(\frac{\varpi_{1} V_{V}(0)^{\left(1-b_{V}\right) / 2}+\varpi_{2}}{\varpi_{2}}\right)
$$

It can be seen that the transformed error $\xi_{V}(t)$ converges to the reference signal in finite-time $T_{V, 1}+T_{V, 2}$ in spite of lumped disturbances, which guarantees the transient and steady-state performance of airspeed tracking error within constrained fixed-time performance envelops.

This completes the proof.

Remark 7: According to the design process of the control scheme in this paper, the parameters of the controller are divided into fixed-time performance functions, robust exact first-order differentiators, disturbance observers, and backstepping control. The parameters $\sigma_{h, 0}, \sigma_{V, 0}, \sigma_{h, \infty}, \sigma_{V, \infty}$ of the fixed-time performance functions are selected according to the initial values and the required steady-state error limits of the altitude/airspeed tracking errors. The specified convergence times $T_{h}, T_{V}$ can be selected based on the control requirements and the actual flight performance limits of the aircraft. The parameters selection rules of robust exact first-order differentiators and disturbance observers can refer to Section 2.2 and Theorem 1. It is worth noting that large parameters can accelerate the convergence rate, but excessively large parameters may cause the chattering problem of the differentiators and disturbance observers. Therefore, the parameters of the differentiators and disturbance observers need to balance the convergence rate and the chattering problem, that is, under the premise of meeting the convergence rate, the differentiators and disturbance observers select small parameters. The parameters $\eta_{1}, \eta_{2}, \eta_{3}, \eta_{4}$ of the backstepping control increases sequentially, and the large parameters can accelerate the convergence rate of the system. Generally, the smaller of the specified convergence time $T_{h}$, the larger $\eta_{1}, \eta_{2}, \eta_{3}, \eta_{4}$. The parameters $\beta_{i}, \lambda_{i}, i=1,2,3,4$ are generally small, and large parameters will cause chattering problem. The parameters $0<a, b<1$ are generally large, and small $a, b$ will cause chattering problem. The larger the parameters $\eta_{V, 1}, \eta_{V, 2}$ are selected of airspeed controller, the faster the convergence rate of the airspeed closedloop system. Generally, the smaller of the specified convergence time $T_{V}$, the larger $\eta_{V, 1}, \eta_{V, 2}$.

\section{Simulation results}

In this section. two numerical simulations are presented to verify the effectiveness and robustness of the proposed backstepping controller with fixed-time preseribed performance (FTPPBC). The nonlinear longitudinal model of fixed wing UAV with model uncertainties and external disturbances is taken as the simulation object. The main geometric characteristics of the fixed wing UAV and the values of the aerodynamic coefficients are shown in Z. Yu et al. (2018). Based on the basic parameters of the fixed wing UAV, the simulation initial conditions are set as $h=292 \mathrm{~m}, V=151 \mathrm{~m} / \mathrm{s}, \gamma=0^{\circ}, \alpha=1.885^{\circ}, q=0 \mathrm{rad} / \mathrm{s}$. The reference altitude command is $h_{c}=300 \mathrm{~m}$, and the reference airspeed command is $V_{c}=150 \mathrm{~m} / \mathrm{s}$. Furthermore, in order to verify the robustness of the proposed control scheme, the mass of UAV is applied with $5 \%$ uncertainty, and the lift coefficient, drag coefficient and pitching moment coefficient are applied with $-10 \%, 10 \%, 30 \%$ uncertainties, respectively. 
In addition, the time-varying external disturbances of lift, drag and pitch torque are added as follows:

$$
\left[\begin{array}{l}
\Delta L \\
\Delta D \\
\Delta M
\end{array}\right]=\left[\begin{array}{l}
8000[\sin (1.2 t)+0.1 \cos (t)] \\
1000[\sin (0.8 t)+0.2 \cos (1.2 t)] \\
5000[0.3 \sin (1.6 t)+\cos (0.8 t)]
\end{array}\right]
$$

\subsection{Performance comparison with the existing control method}

In this numerical simulation, comparison studies are carried out against the active disturbance rejection control-based prescribed performance backstepping controller (ADRC-PPBC) proposed in Yang, Tan, and Yue (2020). In Yang et al. (2020), the extended state observers are utilized to estimate and compensate for uncertainties, the tracking differentiators are employed to obtain the differential signals, and the auxiliary system is used to overcome the input saturation constrain in the controller.

For FTPPBC, the parameters of the novel fixed-time performance functions for altitude and airspeed tracking control are set as $\sigma_{h, 0}=10, \sigma_{h, \infty}=0.1, T_{h}=$ $2.5 \mathrm{~s}, \delta_{h, \text { min }}=1, \delta_{h, \text { max }}=1, \sigma_{V, 0}=1.2, \sigma_{V, \infty}=0.02, T_{V}=1 \mathrm{~s}, \delta_{V, \text { min }}=1, \delta_{V, \text { max }}=1$. The parameters of disturbance observers are set as $r_{2,1}=r_{4,1}=r_{V, 1}=1$, $r_{2,2}=r_{4,2}=r_{V, 2}=0.5, r_{2,3}=r_{4,3}=r_{V, 3}=0.5, r_{2,4}=r_{4,4}=r_{V, 4}=0.5$, $\tau=0.05, T_{\vartheta}=0.1$. The parameters of robust exact first-order differentiators are set as $\kappa_{2,1}=0.7, \kappa_{2,2}=0.4, \kappa_{2,3}=0.5, \kappa_{2,4}=0.5, \kappa_{3,1}=0.8, \kappa_{3,2}=0.6, \kappa_{3,3}=0.5, \kappa_{3,4}=0.5$, $\kappa_{4,1}=1, \kappa_{4,2}=0.6, \kappa_{4,3}=0.5, \kappa_{4,4}=0.5, \tau_{0}=0.1, T_{\vartheta_{0}}=0.1$. The parameters of backstepping controller and error compensation mechanism are chosen as $\eta_{1}=1.1, \eta_{2}=3.3$, $\eta_{3}=10, \eta_{4}=30, \beta_{1}=\beta_{2}=\beta_{3}=\beta_{4}=0.1, \lambda_{1}=\lambda_{2}=\lambda_{3}=\lambda_{4}=0.1, a=0.8, b=0.9$. The parameters of airspeed controller are chosen as $\eta_{V, 1}=0.3, \eta_{V, 2}=0.3$.

The simulation results of two control schemes are shown in Figs. 6-15. Fig. 6 and Fig. 8 show the curves of the altitude and airspeed tracking. The response curves of the altitude and airspeed tracking errors are sketched in Fig. 7 and Fig. 9, from which it is evident that the altitude and airspeed tracking errors under ADRC-PPBC and FTPPBC are limited to respective performance envelops with small overshoot. The altitude and airspeed tracking errors under ADRC-PPBC converge to steady-state error ranges $(-0.1 \mathrm{~m}, 0.1 \mathrm{~m})$ and $(-0.02 \mathrm{~m} / \mathrm{s}, 0.02 \mathrm{~m} / \mathrm{s})$ in $3.5 \mathrm{~s}$ and $3.3 \mathrm{~s}$, respectively. The altitude and airspeed tracking errors under the proposed FTPPBC are finally converge to the prescribed steady-state error ranges $(-0.1 \mathrm{~m}, 0.1 \mathrm{~m})$ and $(-0.02 \mathrm{~m} / \mathrm{s}, 0.02 \mathrm{~m} / \mathrm{s})$ in preassigned fixed-time $2.5 \mathrm{~s}$ and $1 \mathrm{~s}$. Therefore, due to the fixed-time prescribed performance, FTPPBC can reach the control command in arbitrarily preassigned fixedtime, which has better convergence performance than ADRC-PPBC. Meanwhile, the other system states i.e., flight-path angle $\gamma$, angle of attack $\alpha$, and pitch rate $q$, illustrated in Figs. 10-12, are always within the admissible bounded ranges. Fig. 13 shows the comparison curves between the actual disturbances and the disturbances estimates of the longitudinal model. It can be seen from Fig. 13 that the disturbance observers estimate the disturbances accurately. Based on the disturbance observer, the proposed FTPPBC presents excellent robustness against model uncertainties and external disturbances. Moreover, the control inputs of thrust throttle setting and elevator deflection in Figs. 14-15 are continuous and smooth, therefore, the control inputs of proposed FTPPBC are easy to implement in practice. 


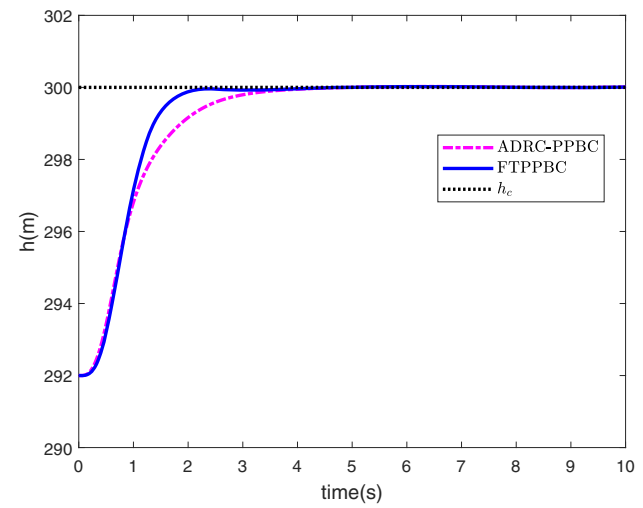

Figure 6. Comparison results in altitude tracking.

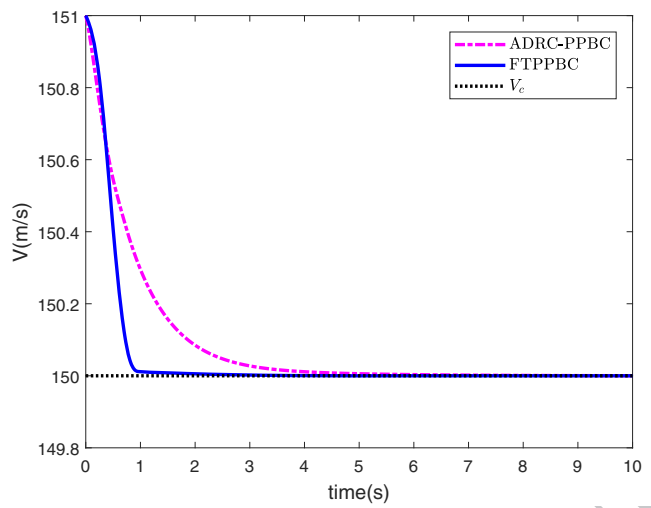

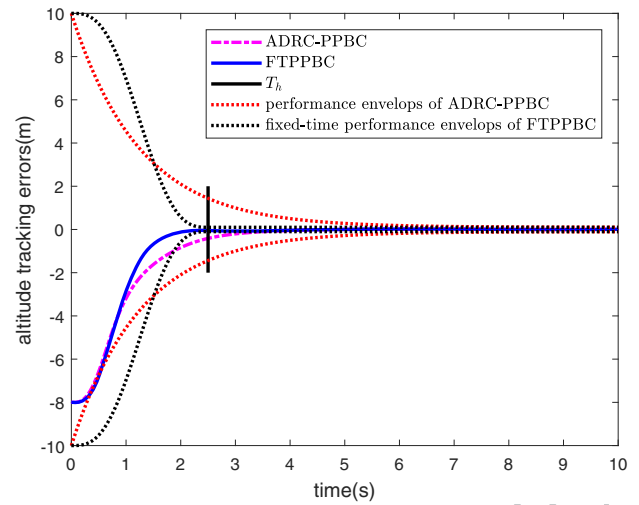

Figure 7. Comparison results in altitude tracking errors.

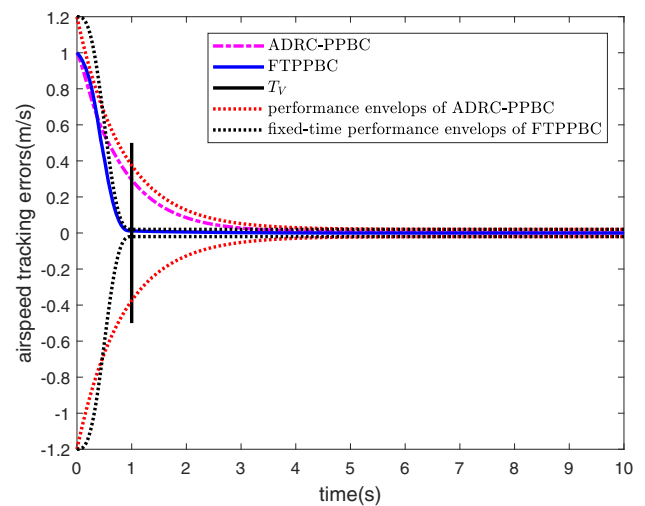

Figure 8. Comparison results in airspeed tracking. Figure 9. Comparison results in airspeed tracking errors.



Figure 10. The curves of flight-path angle.

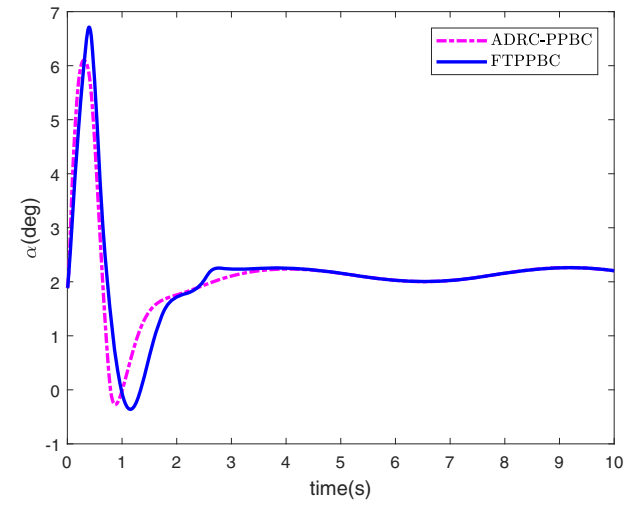

Figure 11. The curves of angle of attack. 


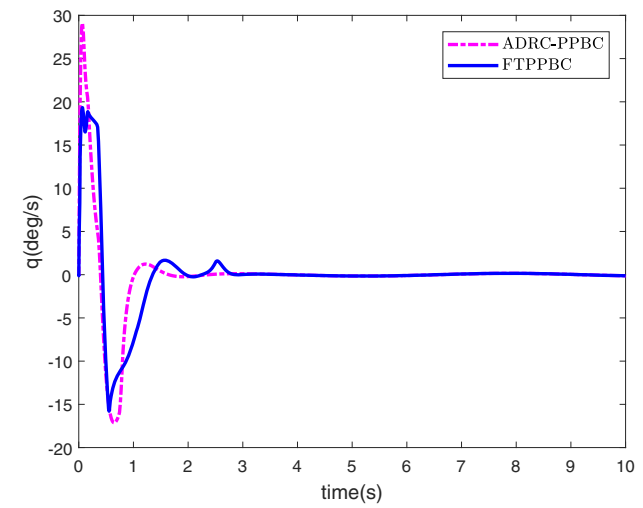

Figure 12. The curves of pitch rate.

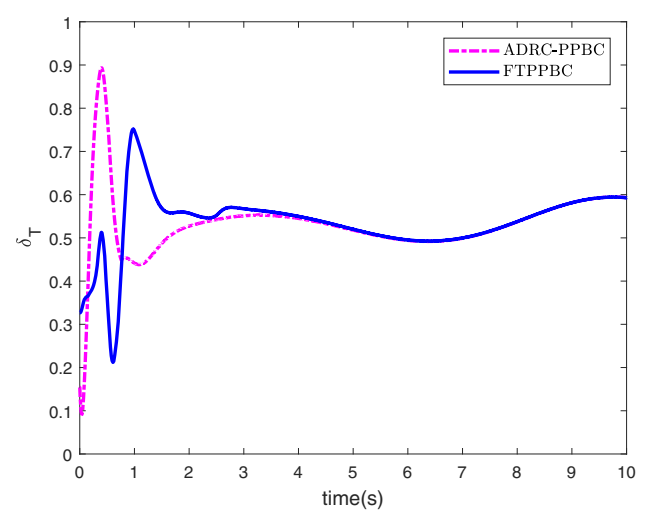

Figure 14. The curves of thrust throttle setting.

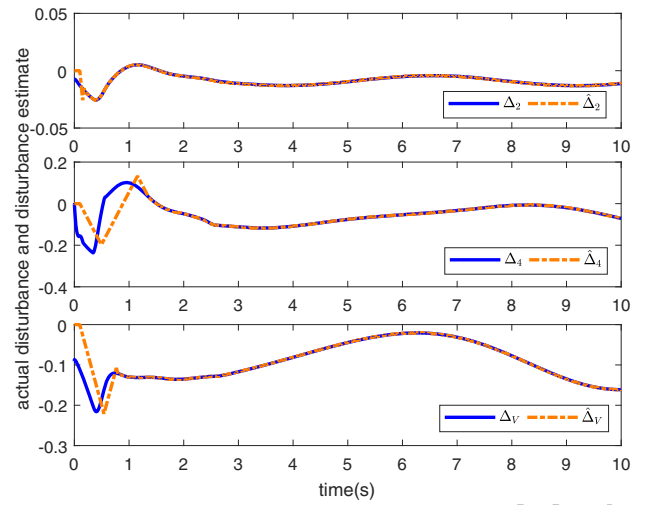

Figure 13. Disturbance estimate curves of the proposed FTPPBC.

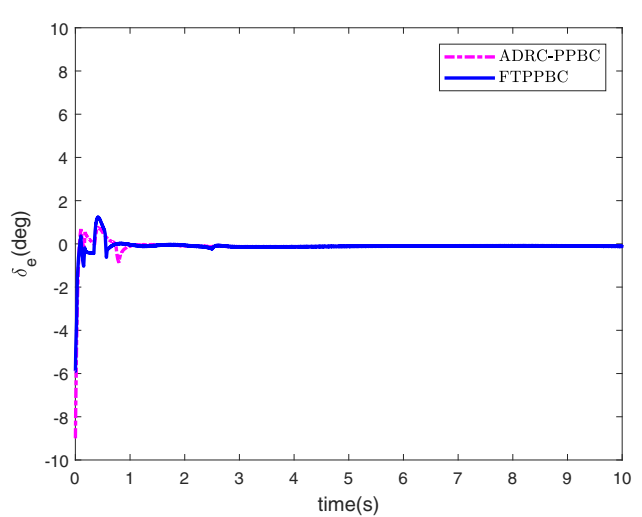

Figure 15. The curves of elevator deflection.

\subsection{Comparison results with conventional PPBC}

In this numerical simulation, comparison studies are carried out against the designed FTPPBC with conventional prescribed performance backstepping controller (PPBC). The backstepping controller of PPBC is the same as FTPPBC. The conventional exponentially decaying performance functions of PPBC are chosen as follows:

$$
\begin{aligned}
& \sigma_{h}(t)=\left(\sigma_{h, 0}-\sigma_{h, \infty}\right) e^{-l_{h} t}+\sigma_{h, \infty} \\
& \sigma_{V}(t)=\left(\sigma_{V, 0}-\sigma_{V, \infty}\right) e^{-l_{V} t}+\sigma_{V, \infty}
\end{aligned}
$$

where $\sigma_{h, 0}=10, \sigma_{h, \infty}=0.1, \sigma_{V, 0}=1.2, \sigma_{V, \infty}=0.02$ are chosen the same as fixed-time performance functions in Section 6.1. The exponents $l_{h}, l_{V}$ are set as $l_{h}=0.3, l_{V}=0.6$ for $\mathrm{PPBC} 1, l_{h}=0.5, l_{V}=1$ for $\mathrm{PPBC} 2, l_{h}=0.7, l_{V}=1.4$ for PPBC $3, l_{h}=0.9, l_{V}=$ 1.8 for PPBC 4, respectively. The other parameters and initial states in PPBC 1-4 are set the same as FTPPBC in Section 6.1.

The simulation results of two control schemes are shown in Figs. 16-24. As can be seen from Figs. 16-19, the altitude and airspeed tracking errors under FTPPBC evolve strictly inside respective performance envelops, and finally converge to the prescribed steady-state error ranges $(-0.1 \mathrm{~m}, 0.1 \mathrm{~m})$ and $(-0.02 \mathrm{~m} / \mathrm{s}, 0.02 \mathrm{~m} / \mathrm{s})$ in pre- 
assigned fixed-time 2.5s and 1s. As the exponents $l_{h}, l_{V}$ increase, illustrated in Figs. 17, the altitude tracking errors under PPBC 1-4 converge to steady-state error range $(-0.1 \mathrm{~m}, 0.1 \mathrm{~m})$ in $3.7 \mathrm{~s}, 3.3 \mathrm{~s}, 2.9 \mathrm{~s}, 2.7 \mathrm{~s}$, respectively, and the airspeed tracking errors under PPBC 1-4 converge to steady-state error range $(-0.02 \mathrm{~m} / \mathrm{s}, 0.02 \mathrm{~m} / \mathrm{s})$ in $3.0 \mathrm{~s}$, $2.5 \mathrm{~s}, 2.1 \mathrm{~s}, 1.9 \mathrm{~s}$, respectively. It is worth noting that the relationship between $l_{h}, l_{V}$ and actual convergence time is nonlinear, and when the simulation conditions and parameters change, the convergence time also changes, that is, it is difficult to ensure the convergence time by selecting specified $l_{h}, l_{V}$. Therefore, for actual control problems, FTPPBC can directly specify the convergence time, which is more convenient than the conventional PPBC to select appropriate $l_{h}, l_{V}$ through a series of simulations. As illustrated in Figs. 20-22, the flight-path angle $\gamma$, angle of attack $\alpha$, and pitch rate $q$ under PPBC 1-4 and FTPPBC are always within the admissible bounded ranges. Since the UAV is in the flight phase, the idle throttle is 0.05 , that is, $\delta_{T} \in[0.05,1]$. As can be seen from Figs. 23-24, the thrust throttle setting and elevator deflection of FTPPBC are not saturation, while the thrust throttle settings of PPBO 1-4 are saturation at the initial time. According to the simulation results and the analysis in Section 3.3, compared with PPBC, FTPPBC can effectively avoid control input saturation at the initial time.

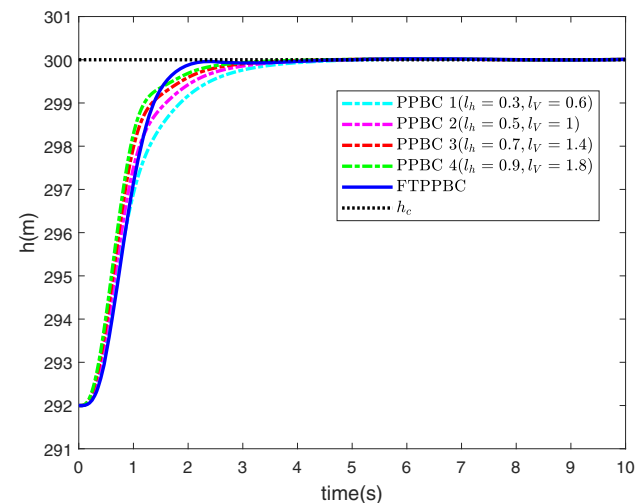

Figure 16. Comparison results in altitude tracking.

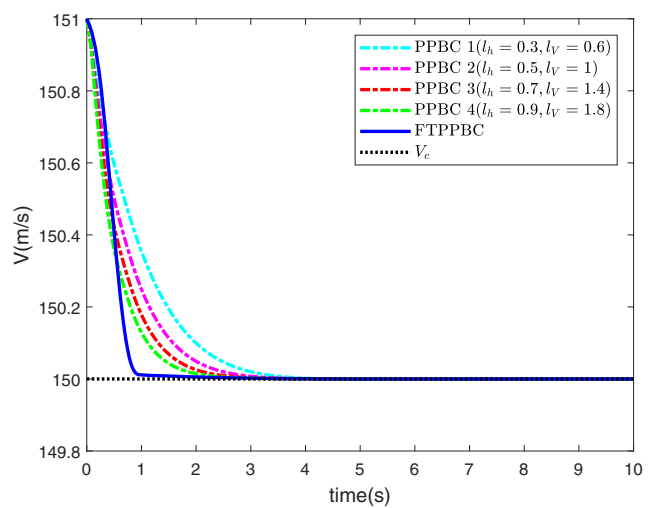

Figure 18. Comparison results in airspeed tracking.

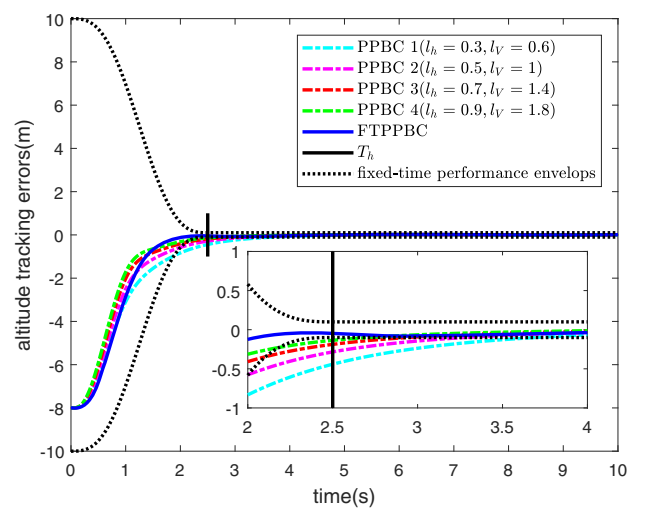

Figure 17. Comparison results in altitude tracking errors.

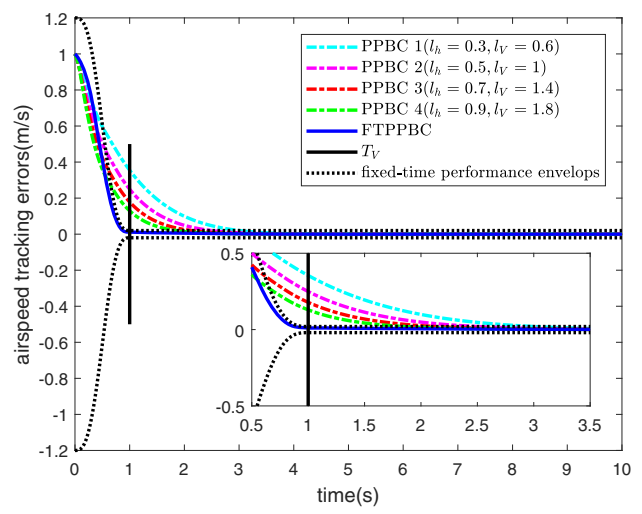

Figure 19. Comparison results in airspeed tracking errors. 


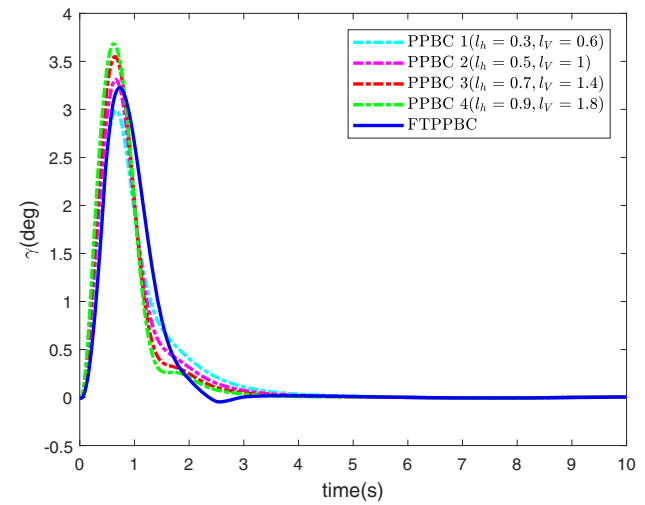

Figure 20. The curves of flight-path angle.

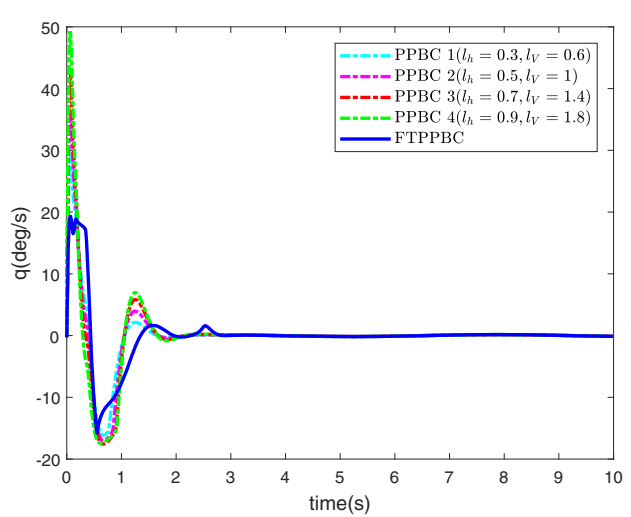

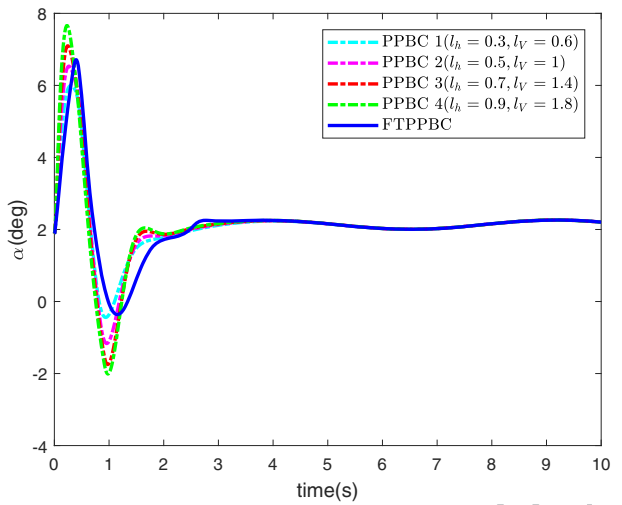

Figure 21. The curves of angle of attack.

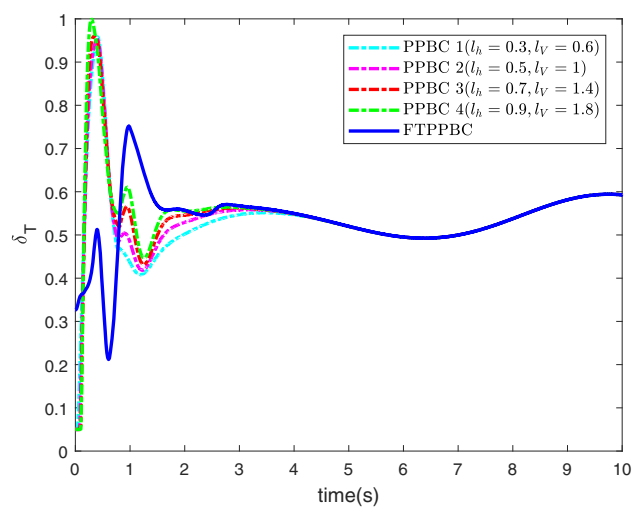

Figure 22. The curves of pitch rate.

Figure 23. The curves of thrust throttle setting.

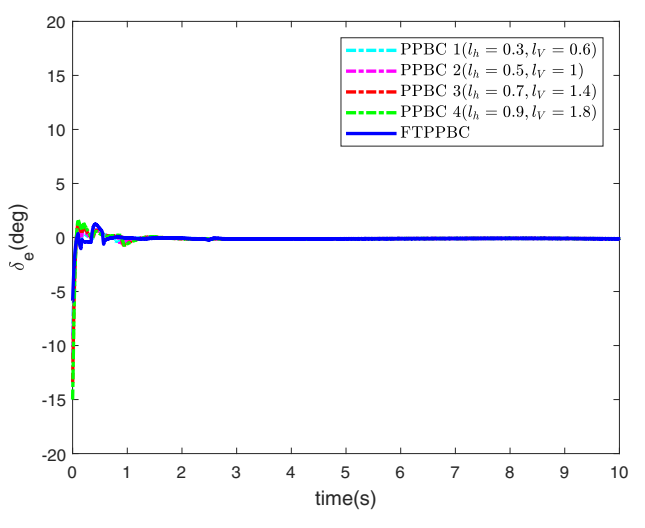

Figure 24. The curves of elevator deflection.

\section{Conclusion}

In this paper, we aim at achieving arbitrarily fixed-time prescribed performance control for the longitudinal model of fixed wing UAV subject to model uncertainties and external disturbances. A novel performance function with arbitrarily preassigned fixed-time convergence property is developed, which imposes priori performance en- 
velops on both altitude and airspeed tracking errors. Furthermore, based on the disturbance observer, a novel backstepping control scheme with modified error compensation mechanism is proposed to guarantee altitude tracking error within constrained fixed-time performance envelops in spite of lumped disturbances, whereas airspeed controller with fixed-time prescribed performance is designed. Finally, numerical simulations are used to verify the effectiveness of the proposed control scheme. In the future work, the coordinated trajectory tracking control with fixed-time prescribed performance of multiple UAVs will be explored.

\section{Disclosure statement}

No potential conflict of interest was reported by the authors.

\section{Funding}

This work was supported by GDAS' Project of Science and Technology Development (2018GDASCX-0202, 2018GDASCX-0804).

\section{References}

Al Younes, Y., Drak, A., Noura, H., Rabhi, A., \& El Hajjaji, A. (2016). Robust model-free control applied to a quadrotor uav. Journal of Intelligent \&3 Robotic Systems, 84(1-4), $37-52$.

Angulo, M. T., Moreno, J. A., \& Fridman, D. (2013). Robust exact uniformly convergent arbitrary order differentiator. Automatica, $49(8), 2489-2495$.

Back, J., \& Kim, J.-S. (2015). A disturbance observer based practical coordinated tracking controller for uncertain heterøgeneous multi-agent systems. International Journal of Robust and Nonlinear Control, 25(14), 2254-2278.

Bechlioulis, C. P., Doulgeri, Z., \& Rovithakis, G. A. (2012). Guaranteeing prescribed performance and contact maintenance via an approximation free robot force/position controller. Automatica, 48(2), 360-365.

Bechlioulis, C. P. \& Rovithakis, G. A. (2008). Robust adaptive control of feedback linearizable mimo nonlinear systems with prescribed performance. IEEE Transactions on Automatic Control, 53(9), 2090-2099.

Bechlioulis, C. P. \& Rovithakis, G. A. (2014). A low-complexity global approximation-free control scheme with prescribed performance for unknown pure feedback systems. Automatica, 50(4), 1217-1226.

Bechlioulis, C. P., \& Rovithakis, G. A. (2016). Decentralized robust synchronization of unknown high order nonlinear multi-agent systems with prescribed transient and steady state performance. IEEE Transactions on Automatic Control, 62(1), 123-134.

Besnard, L., Shtessel, Y. B., \& Landrum, B. (2012). Quadrotor vehicle control via sliding mode controller driven by sliding mode disturbance observer. Journal of the Franklin Institute, 349(2), 658-684.

$\mathrm{Bu}, \mathrm{X}$. (2018). Guaranteeing prescribed performance for air-breathing hypersonic vehicles via an adaptive non-affine tracking controller. Acta Astronautica, 151, 368-379.

Bu, X., Wu, X., Huang, J., \& Wei, D. (2016). Robust estimation-free prescribed performance back-stepping control of air-breathing hypersonic vehicles without affine models. International Journal of Control, 89(11), 2185-2200. 
Bu, X., Wu, X., Zhang, R., Ma, Z., \& Huang, J. (2015). Tracking differentiator design for the robust backstepping control of a flexible air-breathing hypersonic vehicle. Journal of The Franklin Institute-engineering and Applied Mathematics, 352(4), 1739-1765.

Castañeda, H., Salas-Peña, O. S., \& de León-Morales, J. (2017). Extended observer based on adaptive second order sliding mode control for a fixed wing uav. ISA transactions, 66, $226-232$.

Chen, F., Jiang, R., Zhang, K., Jiang, B., \& Tao, G. (2016). Robust backstepping slidingmode control and observer-based fault estimation for a quadrotor uav. IEEE Transactions on Industrial Electronics, 63(8), 5044-5056.

Espitia, N., Polyakov, A., Efimov, D., \& Perruquetti, W. (2019). Boundary time-varying feedbacks for fixed-time stabilization of constant-parameter reaction-diffusion systems. Automatica, 103(103), 398-407.

Farrell, J. A., Polycarpou, M., Sharma, M., \& Dong, W. (2009). Command filtered backstepping. IEEE Transactions on Automatic Control, 54(6), 1391-1395.

Gao, Z., \& Guo, G. (2019). Command-filtered fixed-time trajectory tracking control of surface vehicles based on a disturbance observer. International Journal of Robust and Nonlinear Control, 29(13), 4348-4365.

Gao, Z., \& Guo, G. (2020). Fixed-time sliding mode formation control of auvs based on a disturbance observer. IEEE/CAA Journal of Automatica Sinica, 7(2), 539-545.

Guo, Q., Zhang, Y., Celler, B. G., \& Su, S. W. (2019). Neural adaptive backstepping control of a robotic manipulator with prescribed performance constraint. IEEE Transactions on Neural Networks, 30(12), 3572-3583.

Haghighi, D. A., \& Mobayen, S. (2018). Design of an adaptive super-twisting decoupled terminal sliding mode control scheme for a class of fourth-order systems. Isa Transactions, 75, 216-225.

Hua, C., Chen, J., \& Guan, X. (2018). Adaptive prescribed performance control of quavs with unknown time-varying payload and wind gust/disturbance. Journal of the Franklin Institute, 355(14), 6323-6338.

Kalliny, A. N., Elbadawy, A. A., \& Elkhamisy, S. M. (2018). Command-filtered integral backstepping control of longitudinal flapping-wing flight. Journal of Guidance Control and Dynamics, 41(7), 1556-1568.

Kostarigka, A. K., Doulgeri, Z. \& Rovithakis, G. A. (2013). Prescribed performance tracking for flexible joint robots with unknown dynamics and variable elasticity. Automatica, 49(5), $1137-1147$.

Levant, A. (2003). Higher-order sliding modes, differentiation and output-feedback control. International journal of Control, 76 (9-10), 924-941.

Li, Y., \& Tong, S. (2016). Command-filtered-based fuzzy adaptive control design for mimoswitched nonstrict-feedback nonlinear systems. IEEE Transactions on Fuzzy Systems, $25(3), 668-681$.

Li, Y., \& Tong, S. (2018). Adaptive fuzzy control with prescribed performance for blocktriangular-structured nonlinear systems. IEEE Transactions on Fuzzy Systems, 26(3), $1153-1163$

Lu, H., Liu, C., Guo, L., \& Chen, W.-H. (2015). Flight control design for small-scale helicopter using disturbance-observer-based backstepping. Journal of Guidance, Control, and Dynamics, 38(11), 2235-2240.

Mobayen, S. (2015). Finite-time tracking control of chained-form nonholonomic systems with external disturbances based on recursive terminal sliding mode method. Nonlinear Dynamics, 80(1), 669-683.

Mobayen, S., \& Ma, J. (2018). Robust finite-time composite nonlinear feedback control for synchronization of uncertain chaotic systems with nonlinearity and time-delay. Chaos Solitons and Fractals, 114, 46-54.

Mofid, O., \& Mobayen, S. (2018). Adaptive sliding mode control for finite-time stability of quad-rotor uavs with parametric uncertainties. ISA transactions, 72, 1-14.

Nikdel, N., Badamchizadeh, M., Azimirad, V., \& Nazari, M. A. (2016). Fractional-order 
adaptive backstepping control of robotic manipulators in the presence of model uncertainties and external disturbances. IEEE Transactions on Industrial Electronics, 63(10), 62496256.

Pan, Y., Wang, H., Li, X., \& Yu, H. (2018). Adaptive command-filtered backstepping control of robot arms with compliant actuators. IEEE Transactions on Control Systems and Technology, 26(3), 1149-1156.

Peydayesh, A., Arefi, M. M., \& Modares, H. (2018). Distributed neuro-adaptive control protocols for non-strict feedback non-linear mass with input saturation. IET Control Theory \& Applications, 12(11), 1611-1620.

Polyakov, A. (2012). Nonlinear feedback design for fixed-time stabilization of linear control systems. IEEE Transactions on Automatic Control, 57(8), 2106-2110.

Polyakov, A., Efimov, D., \& Brogliato, B. (2019). Consistent discretization of finite-time and fixed-time stable systems. Siam Journal on Control and Optimization, 57(1), 78-103.

Poultney, A., Gong, P., \& Ashrafiuon, H. (2019). Integral backstepping control for trajectory and yaw motion tracking of quadrotors. Robotica, 37(2), 300-320.

Pukdeboon, C., \& Siricharuanun, P. (2014). Nonsingular terminal sliding mode based finitetime control for spacecraft attitude tracking. International Journal of Control, Automation and Systems, 12(3), 530-540.

Qiao, H., Meng, H., Wang, M., Ke, W., \& Sun, J. (2019). Adaptive control for hypersonic vehicle with input saturation and state constraints. Aerospace Science and Technology, 84, 107-119.

Qiu, Y., Liang, X., Dai, Z., Cao, J., \& Chen, Y. (2015). Backstepping dynamic surface control for a class of non-linear systems with time-varying output constraints. IET Control Theory \& Applications, 9(15), 2312-2319.

Sun, Z., Yun, M., \& Li, T. (2017). A new approach to fast global finite-time stabilization of high-order nonlinear system. Automatica, 81(81), 455-463.

Swaroop, D., Hedrick, J. K., Yip, P. P., \& Gerdes, J. C. (2000). Dynamic surface control for a class of nonlinear systems. IEEE transactions on automatic control, 45(10), 1893-1899.

Tong, S., Sui, S., \& Li, Y. (2014). Fuzzy adaptive output feedback control of mimo nonlinear systems with partial tracking errors constrained. IEEE Transactions on Fuzzy Systems, $23(4), 729-742$.

Tooranjipour, P., Vatankhah, R., \& Arefi, M. M. (2019). Prescribed performance adaptive fuzzy dynamic surface control of nonaffine time-varying delayed systems with unknown control directions and dead-zone input. International Journal of Adaptive Control and Signal Processing, 33(7), 1134-1156.

Wang, X., Yu, X., Li, S., \& Liu, J. (2019). Composite block backstepping trajectory tracking control for disturbed unmanned helicopters. Aerospace Science and Technology, 85, 386398.

Xi, X., Mobayen, S., Ren, H., \& Jafari, S. (2018). Robust finite-time synchronization of a class of chaotic systems via adaptive global sliding mode control:. Journal of Vibration and Control, 24(17), 3842-3854.

Yang, Y., Tan, J., \& Yue, D. (2020). Prescribed performance tracking control of a class of uncertain pure-feedback nonlinear systems with input saturation. IEEE Transactions on Systems, Man, and Cybernetics: Systems, 50(5), 1733-1745.

Yu, J., Shi, P., Dong, W., \& Yu, H. (2015). Observer and command-filter-based adaptive fuzzy output feedback control of uncertain nonlinear systems. IEEE Transactions on Industrial Electronics, 62(9), 5962-5970.

Yu, J., Shi, P., \& Zhao, L. (2018). Finite-time command filtered backstepping control for a class of nonlinear systems. Automatica, 92, 173-180.

Yu, J., Zhao, L., Yu, H., Lin, C., \& Dong, W. (2017). Fuzzy finite-time command filtered control of nonlinear systems with input saturation. IEEE transactions on cybernetics, $48(8)$, 2378-2387.

Yu, Z., Qu, Y., \& Zhang, Y. (2018). Safe control of trailing uav in close formation flight against actuator fault and wake vortex effect. Aerospace Science and Technology, 77, 189-205. 
Zhang, J., Xia, J., Sun, W., Wang, Z., \& Shen, H. (2019). Command filter-based finite-time adaptive fuzzy control for nonlinear systems with uncertain disturbance. Journal of The Franklin Institute-engineering and Applied Mathematics, 356(18), 11270-11284.

Zhang, Y., Wang, S., Chang, B., \& Wu, W. (2019). Adaptive constrained backstepping controller with prescribed performance methodology for carrier-based uav. Aerospace Science and Technology, 92, 55-65.

Zuo, Z. (2015). Nonsingular fixed-time consensus tracking for second-order multi-agent networks. Automatica, 54, 305-309. 


\title{
Backstepping control with fixed-time prescribed performance for fixed wing UAV under model uncertainties and external disturbances
}

\author{
Tan, Jian
}

Taylor and Francis

Tan J, Guo S. (2022) Backstepping control with fixed-time prescribed performance for fixed wing UAV under model uncertainties and external disturbances. International Journal of Control, Volume 95, Issue 4, 2022, pp. 934-951

https://doi.org/10.1080/00207179.2020.1831700

Downloaded from Cranfield Library Services E-Repository 